\title{
Single-Cell Transcriptome Analysis Reveals Heterogeneity and Convergence of the Tumor Microenvironment in CRC
}

\section{Siyuan Xie}

Zhejiang University School of Medicine Second Affiliated Hospital

\section{Yangke Cai}

Zhejiang University School of Medicine Second Affiliated Hospital

Delong Chen

Zhejiang University School of Medicine Second Affiliated Hospital

\section{Yu Xiang}

Huzhou Central Hospital

\section{Wen Cai}

Zhejiang University School of Medicine Second Affiliated Hospital

\section{Bin Yang}

Zhejiang University School of Medicine Second Affiliated Hospital

\section{Jianshan Mao}

Zhejiang University School of Medicine Second Affiliated Hospital Jun Ye ( $\nabla$ wzmcyejun@zju.edu.cn )

The Second Affiliated Hospital, Zhejiang University School of Medicine

\section{Research}

Keywords: scRNA-seq, colorectal cancer, CIBERSORTx, prognosis

Posted Date: August 31st, 2021

DOI: https://doi.org/10.21203/rs.3.rs-836574/v1

License: (c) (1) This work is licensed under a Creative Commons Attribution 4.0 International License. Read Full License 


\section{Abstract}

Background: Colorectal cancer (CRC) ranks second for mortality and third for morbidity among the most commonly diagnosed cancers worldwide. Tumor microenvironment (TME) has been reported to correlate with CRC outcomes and treatment efficacy. Single-cell RNA sequencing (scRNA-seq) is an indispensable tool for unravelling cellular heterogeneity and deconstructing tissues into cell types, offering enormous potential for new discoveries.

Methods: The single-cell RNA-seq profiles included 10,398 cells from 10 human CRC samples (accession number GSE146771), which were obtained from the Gene Expression Omnibus (GEO, http://www.ncbi.nlm.nih.gov/geo/) database. We identified 8 major cell types and 25 subgroups of cells derived from tumors, para-tumors, and peripheral blood to investigate the intratumoral heterogeneity and convergence of CRC.

Results: This study established the heterogeneity, convergence, and differentiation trajectory of immune cells and stromal cells within CRC tissues. We also identified the terminal stage of each cell type of metabolism remodeling and immunosuppressive phenotypes. SCENIC analysis revealed the potential targets of transcriptional factors to reverse this process. Based on the cell subgroup fractions calculated by CIBERSORTx and the clinical data in The Cancer Genome Atlas (TCGA), we constructed a new prognosis model of CRC.

Conclusions: This study provides a new perspective for understanding the heterogeneity and convergence of the TME and will aid the development of immunotherapies and prognosis of CRC patients.

\section{Background}

Colorectal cancer is the third most common cancer worldwide and ranks second for mortality; it caused over 935,000 deaths in 2020 worldwide ${ }^{58}$. Due to population growth and aging, by 2035 , the number of deaths is expected to rise in all countries for colon and rectal cancers by $60.0 \%$ and $71.5 \%$, respectively ${ }^{3}$. Traditional treatments for primary and metastatic colorectal cancer include laparoscopic surgery for primary disease, resection of the metastatic part, radiotherapy, and chemotherapy ${ }^{15}$. However, the clinical prognosis remains unsatisfactory. New immunotherapy treatments and targeted therapies have rapidly become established as major treatment modes for many types of solid cancers, such as colorectal cancer. Cancer immunotherapies, including checkpoint inhibitors and adoptive cell therapy, oncolytic viruses, and cancer vaccines manipulate the immune system to recognize and attack cancer cells. Molecular targeted therapies interfere with specific molecules to block cancer growth, progression, and metastasis, such as HER2-targeted therapy in breast cancer ${ }^{44}$, EGFR-targeted therapy in lung cancer ${ }^{27}$, and BRAF-targeted therapy in malignant melanoma ${ }^{2}$.

The tumor microenvironment (TME) comprises various cell types: tumor cells, immune cells, stromal cells, and extracellular components such as growth factors, cytokines, extracellular matrix, and hormones 
surrounding the cancer cells. Cancer cells have been studied extensively to understand tumor heterogeneity. However, immune cells and stromal cells have also demonstrated heterogeneity ${ }^{47} \cdot \operatorname{CD} 8+T$ cells are classically subdivided into naïve T cells, effector T cells, and subsets of memory $T$ cells according to their differentiation state ${ }^{62}$. Macrophages have been classically divided into two groups; M1 and M2 macrophages. More recently, additional macrophage phenotypes such as $\mathrm{M}(\mathrm{Hb})$, Mox, and M4 have emerged ${ }^{30}$. We identified three mainly unique $C D 8+T$ cell subsets in the $C R C$, including naïve $T$ cells, T effector cells, and exhausted CD8 T cells (Tex). Macrophages show extreme heterogeneity and can be polarized into $\mathrm{M} 1$ and $\mathrm{M} 2$ depending on their environment. We also detected two fibroblast subtypes in our study.

There has been much effort put into developing new therapeutic strategies to target the TME more efficiently. Tumor-associated macrophages (TAMs) and cancer-associated fibroblasts (CAFs) were reported as potential targets for cancer therapies in previous studies ${ }^{56,59}$. However, the TME is highly complex and heterogeneous and understanding the major events occurring in the TME that support primary tumor growth and how these events impact the modulation of the environment are vital. These novel treatment strategies require a deeper understanding of intratumoral heterogeneity and the heterogeneity of cells in the TME during differentiation. We observed that different cell types always exhibit a common phenotype that promotes tumor progression in the TME. For example, we noticed that Tex, TAM, and CAF jointly present enhanced lipid metabolism, and increased expression of inhibitory cytokines and inhibitory immune checkpoints, which accelerate the construction of the tumor immunosuppressive microenvironment. We supposed that finding new common and highly expressed immune checkpoints or transcription factors in these different cell types might represent important targets for treating CRC.

Immune checkpoint blockade (ICB) can disrupt immune surveillance by cancer cells and has dramatically altered cancer treatment paradigms. The programmed cell death 1 (PD-1) receptor and cytotoxic $T$ lymphocyte-associated protein 4 (CTLA-4) have emerged as the dominant inhibitors that regulate T cell responses in colorectal cancer ${ }^{13,68}$. However, they have only been effective in patients with metastatic $\mathrm{CRC}$ that is mismatch-repair-deficient and microsatellite instability-high (dMMR-MSI-H) ${ }^{9}$. Therefore, exploring the relationship between immune checkpoints and microsatellite instability (MSI) and identifying additional inhibitory receptors (IRS) are the foci of our ongoing research.

Single-cell RNA sequencing (scRNA-seq) is an indispensable tool for unravelling cellular heterogeneity and deconstructing tissues into cell types, offering enormous potential for new discoveries ${ }^{21}$. Using scRNA-seq, TME heterogeneity can be discerned. For example, Zhang et al. ${ }^{72}$ performed scRNA-seq on CRC samples, including 10 tumors, para-tumors, and peripheral blood samples. To investigate the intratumoral heterogeneity and convergence in CRC, we performed further bioinformatics analyses based on Zhang's data. The scRNA-seq data were used to identify stochastic variations in gene expression within a single population and reconstruct developmental 'trajectories' to reveal cell fates. This helped us to define key cell populations involved in regulating tumorigenesis and anti-tumor immunity. We observed 
that stromal and immune cells underwent similar metabolic transformations, revealing the heterogeneity and convergence in each cell type in TME and identifying potential targets for CRC therapies.

Furthermore, we constructed a risk model based on the above cells by correlating the scRNA-seq data with the data from the TCGA dataset. Finally, we constructed a new clinical prognostic model incorporating the risktype, the tumor, nodes, and metastasis (TNM) stage, gender, and age. Our work will help to improve clinical treatment strategies and the prognosis for CRC patients.

\section{Results}

We used samples, including tumors, adjacent tumor tissues, and blood from 10 treatment- naïve CRC patients. After quality control (QC) and filtering, as described in Methods, we obtained transcriptomes of 10,398 single cells (Fig. 1B). We performed graph-based clustering using SingleR, an R toolkit for singlecell genomics, to define the major structure of the TME and identified eight cell lineages including CD $4+T$ cells, CD8 + T cells, B cells, myeloid cells, innate lymphoid cells (ILCs), fibroblast cells, endothelial cells, and epithelial cells (Fig. 1A). We also identified 52 different sub-clusters and 25 sub-cell types of each major cell type. We used the markers of each cell subgroup (Figure S1) listed in the CellMarker database and a previous study for this recognition process (refer to Materials and Methods). The top five markers identified by the differences in the main cell lineages were visualized as a bubble plot (Fig. 1C). Since cluster 9 in the CD $4+T$ cells and cluster 8 in the CD $8+T$ cells contained fewer than 30 cells, these clusters were not included in the subsequent analyses. Interestingly, when we traced the tissue origins, it was noted that immune cells, especially Tex, macrophages, TAMs, and dendritic cells (DCs) were highly enriched in tumor tissues. Furthermore, fibroblasts and endothelial cells were also mainly present in tumor tissues, whereas mast cells were mainly present in peripheral blood. We also noticed that most cell clusters were enriched in the tumor and adjacent tumor tissues (Figs. 1A, 1B). To investigate the network of interactions in the TME in CRC, we used CellChat, an R package used to analyze cell-cell communication molecules, to calculate potential ligand-receptor pairs. Network visualization was also performed to visualize the interactions between the many cell types in the TME. Notably, Tex, macrophages, TAMs, and DCs possessed the most interaction pairs with cells from other lineages (Fig. $3 A)$, revealing the dominant role of macrophages in the TME.

\section{CD8+ T cells}

The CD8 + T cells were divided into nine sub-clusters and annotated into four cell types; naïve CD8 T cells, effector memory CD8 T cells, effector CD8 T cells, and Tex (Fig. 2A). To clarify the function of each cell type, we extracted the marker genes and loaded these into the Metascape (http://metascape.org/) (Fig. 2B, C). Pseudotime analysis was applied to construct a differentiation trajectory, and branch expression analysis modeling (BEAM) was used to identify differential gene expression profiles between branches in the trajectory. The pseudotime trajectory revealed that CD8 T cells became exhausted in the TME, and IRS expression increased in a stepwise manner; metabolic remodeling occurred gradually (Fig. 2D, E). We clustered all the transcription factors surrounding the $\mathrm{CD} 8+T$ cells by single-cell regulatory network inference and clustering (SCENIC) analysis and divided them into nine modules using a clustering 
algorithm. Notably, Module 1 transcription factors were significantly activated in Tex (Fig. 3E, F). We speculated that Module 1 transcription factors were significantly related to the process of $\mathrm{T}$ cell exhaustion.

\subsection{Metabolic remodeling in the CD8+ $T$ cell exhaustion process}

Under chronic inflammation such as during cancer, autoimmunity, and chronic infections, T effector cells (Teff) transform into Tex ${ }^{12}$. We identified the differentially expressed genes (DEGs) among the Teff and Tex and performed enrichment analysis (Fig. 2B, C). Compared to Teff, catabolism-related functions and pathways were more enriched in Tex. Tex were enriched in the AMPK pathway (Fig. 2C), which serves as the guardian of metabolism ${ }^{28}$, whereas Teff were not. Besides the different metabolic patterns, there were also major energy metabolism differences between Teff and Tex. The glucose metabolic process was enriched in Teff $(P<0.05)$, while lipid metabolism processes were enriched in Tex, such as lipid biosynthesis and the cholesterol metabolic pathway. Gene Set Variation Analysis (GSVA) analysis demonstrated that, compared to Teff, the lipid and cholesterol metabolic pathways were significantly highly expressed in Tex (Fig. 2H). Interestingly, although Tex appeared in the catabolic pattern, the pathways for lipid and cholesterol biosynthesis were also enriched in Tex. Further pseudotime analysis revealed the $\mathrm{CD} 8+\mathrm{T}$ cells differentiation trajectory. We observed that normal tissue-derived $\mathrm{CD} 8+\mathrm{T}$ cells tended to convert into tumor-infiltrating T cell subgroups, and Tex were present at the end of the differentiation trajectory (Fig. 2E). We extracted genes whose expression increased in the differentiation process and then conducted enrichment analysis. It was not critical whether lipid catabolism occurred as fatty acid beta-oxidation or lipid anabolism, because fatty acid biosynthesis and omega-3, -6 , and -9 fatty acids (FAs) synthesis were all enriched (Fig. 2F). Subsequently, we choose point 1 in the trajectory as the turning point since cells before point 1 were naïve cells. Cells after point 1 were classified into two clusters: cellfate1 and cellfate2. Notably, IRS, including PD-1 and CTLA-4, were highly expressed in cellfate2 (Supplementary Table 1). Since these molecules are markers of Tex, cellfate2 was very much involved in the trajectory leading to the differentiation of exhaustion. BEAM analysis revealed that in cellfate2, genes related to lipid metabolism, such as SREBP, FASN, ACC, HMG-CoA, ACLY, and PPARG were upregulated. These results implied that the differentiation of $T$ cells was related to lipid metabolism remodeling, and abnormal lipid accumulation may be the energy source for Tex.

A previous study showed activation of the PPAR pathway in the metabolic regulation of lipid and lipoprotein levels ${ }^{40}$. Our DEGS analysis and the Pseudotime analysis showed significant enrichment of the PPARG pathway. The GSVA also showed that the PPARG pathway was highly expressed in Tex. Based on these results, we suspect that the lipid metabolism remodeling in Tex is attributed to the activation of the PPARG pathway.

We performed SCENIC analysis to verify this hypothesis and reveal the abnormal transcriptional regulatory network of Tex. Without suspension, PPARG was located in the M1 module. This further 
demonstrates that the PPARG transcription factor may play an important role in lipid reprogramming in Tex. In addition, we also enriched the RORC, NR1D1, and SREBF2 transcription factors in the M1 module, which are also closely associated with lipid metabolism $32,43,70$. For example, SREBF2 is involved in cisplatin resistance in ovarian cancer cells and cholesterol metabolic processes ${ }^{76}$. Our results suggested that transcription factors (TFs) such as PPARG and SREBF2 may participate in the metabolic remodeling in Tex and act as latent targets to reverse this process.

\subsection{Loss of Tex effector function and the DNA damage pathway}

The loss of Tex effector function is classed into three major categories: (1) cell surface IRS upregulated, (2) inhibitory soluble factors and environmental factors such as IL 10 , IL4, TGF- $\beta$, and IFNa/ $\beta$, and (3) immunosuppressive cells ${ }^{34}$.

We examined the immune checkpoints in different cell types (Figure **). Notably, IRS, including TIM3, LAG3, PDCD1 (PD-1), TIGIT, CD27, CTLA-4, and TNFRSF9, were upregulated in Tex. Function enrichment analysis based on the DEGs showed that Teff was enriched in numerous proinflammatory pathways such as the IL-2, $-3,-17$, and -18 signaling pathways, whereas Tex was enriched in IL-4 and -10 immunosuppressive pathways and the PD-1 signaling pathways. GSVA analysis confirmed these results (Fig. 2H).

The pseudotime analysis revealed that genes related to IRS were significantly upregulated, and showed the differentiation of CD8 + T cells, such as PD- 1 and CTLA-4. The expression of immunosuppressiverelated genes such as IL4, IL1RN, and IL4I1 was enhanced, whereas expression of immune activationrelated genes such as IL18BP and IL5RA was reduced. This finding agrees with previous results where T cell exhaustion usually manifests as a stepwise loss of effector functions during persistent infection. Typically, functions such as IL-2, TNF, and IFN-y production are gradually lost ${ }^{66}$.

CellChat analysis was undertaken to determine further the interaction between Tex and other cells in the TME. First, we analyzed the immunosuppressive receptors expressed by Tex, including TIGIT, CTLA-4, ICOS, and PDCD, and found that different cells produced different ligand-receptor modes. Endothelial and tumor cells mainly secreted PVR and NECTIN2, which acted on the TIGIT receptor on the surface of Tex cells. CD80 and CD86 secreted by DCs and TAMs interacted with CTLA. Regulatory T cells (Tregs) mainly secreted CD274 (PD-L1) to act on PDCD1. Furthermore, analysis of the PD-L1 signal pathway regulatory network showed that Tregs were the main senders of PD-L1, with Tex being the main receivers (Fig. 3B).

However, cytokines such as PVR and NECTIN2 also participate in building the tumor immunosuppressive microenvironment. Analysis of the PVR pathway regulatory network showed that tumor and endothelial cells were the main senders, and immunosuppressive cells such as DCs and Tregs were the receivers (Fig. 3D). More interestingly, the fibroblast subgroup served as a mediator in this regulatory network, and this implies that this subgroup could be a potential target for new drugs. The NECTIN2 pathway regulatory network also showed multiple ligand-receptor modes; DCs, endothelial cells, fibroblasts, and TAMs were 
the main senders, and Tregs, Teff, and Tex were the main receivers (Fig. 3C). Because PVR and NECTIN2 can both act on TIGIT, compared to the currently popular PDL1/PDLD1 blockers, TIGIT may not only reverse the exhaustion state of CD8 T cells but may also improve the tumor immunosuppressive microenvironment to a certain extent. Hence, we hypothesized that inhibition of TIGIT could be a new treatment for CRC. Our analyses showed that Tex play major roles in the immunosuppressive microenvironment, and the depletion of CD8 + T cells is an inevitable outcome in the tumor environment.

Interestingly, the BEAM analysis genes indicated in the cellfate2 included IRS receptors and many enriched functional pathways for DNA repair, such as the DNA damage response, DNA replication, and DNA mismatch repair. DEGs enrichment analysis and GSVA also revealed enriched DNA damage pathways in Tex. We suspect that repeated DNA damage and repair is correlated with Tex differentiation and may explain why CRC patients, especially those with MSI-H tumors, are more sensitive to immune checkpoint inhibitors than patients with microsatellite instability-low (MSI-L) tumors ${ }^{46}$. CD $8+$ T cells are likely to differentiate into a terminal differentiation state during the development of T-cell exhaustion, and increased DNA damage and repair leads to an increased frequency of mutations. This explains why CRC patients with MSI-H show a significantly better outcome following immunotherapy.

Finally, we conducted SCENIC analysis to investigate TFs associated with immunity and DNA repair in the M1 module. For example, the zinc finger E-box binding homeobox 1 (ZEB1) inhibits the production of IL-2, which is a $T$ cell growth factor essential for the proliferation of $T$ cells and the generation of effector and memory cells ${ }^{1,31}$. IRF7 is associated with the upregulation of IFNa/ $\beta$ expression. Qinjie Zhou et al ${ }^{78}$. reported that inhibition of IRF7 by AIP, a novel inhibitor, can block the activation of IFN ${ }^{77}$. TRIM28, MYB, and the SOX4 transcription factors are involved in the differentiation of $\mathrm{T}_{\text {cells }} \mathrm{s}^{4}, 20$. Highly expressed transcription factors such as TOPORS, EP300, SOX4, and TFDP1 in Tex are related to DNA damage responses $^{25}, 39,41$. As CRC patients with MSI-H showed significantly better outcomes after immunotherapy, these transcription factors could potentially serve as therapeutic targets to reverse Tex.

\section{Myeloid cells}

Myeloid cells are abundant critical components of the TME. They are a heterogeneous mixture of cell types having both tumor stimulating and suppressing activities. Analysis of the myeloid cells revealed five distinct sub-clusters: monocytes, macrophages, TAMs, DCs, and mast cells (Fig. 4A). Macrophages are a major cell population in the tumor microenvironment; they display high plasticity and perform diverse, supportive functions ${ }^{45}$. Macrophages can be activated and polarized into TAMs by various soluble molecules to promote tumor progression and metastasis. Macrophages and TAMs can be divided into $\mathrm{M} 1$ and $\mathrm{M} 2$ based on their functions; $\mathrm{M} 1$ can secrete proinflammatory cytokines, and M2 exhibit tumor-promoting phenotypes characterized by high levels of immunosuppressive markers and antiinflammatory factors.

Interestingly, when traced back to the tissue source, monocytes were present primarily in the blood, while macrophages and TAMs occurred in most tumor tissues (Fig. 4A). Pseudotime analysis showed that 
monocytes differentiated into macrophages when they entered the TME from the blood and finally differentiated into TAMs (Fig. 4D).

\subsection{TAMs are engaged in constructing the immunosuppressive microenvironment}

Enrichment analysis revealed a mixture of M1 and M2 phenotypes in macrophages and TAMs, but macrophages closely resembled M1-polarized, and TAMs closely resembled M2-polarized. Some pathways associated with M1-like proinflammatory pathways were enriched in macrophages, such as IL$1,-2,-3,-11,-17,-18$, and -21 , the TNF alpha signaling pathway, and the interferon alpha/beta signaling pathway (Fig. 4B). These pathways activate cytokines that promote anti-tumor activities and macrophages that play critical roles in antigen presentation ${ }^{11}$. Macrophages also performed several M2like functions, such as IL-4, -10, and TGF-beta receptor signaling. In contrast, other pathways associated with M2 pro-tumor pathways were rich in TAMs, such as arachidonic acid metabolism, matrix metalloproteinase (MMP), the VEGF signaling pathway, the glucocorticoid receptor pathway, IL-10, -4 and - 13 signaling, and PD-1 signaling. Several M1-like functional pathways were also present, such as interferon gamma signaling and the TNF signaling pathway. However, their expression was dominated by M2-like changes. GSVA analysis also revealed that IFN alpha/beta signaling was enriched in TAMs, while IL-5, -6, -7, and - 17 were enriched in macrophages (Fig. 4C). In conclusion, macrophages mainly display the M1 phenotype, whereas TAMs exhibit M2 phenotypes.

Pseudotime analysis revealed that monocytes converted into macrophages, and finally TAMs (Fig. 4D). In this process, there was altered gene expression of both IL $4 L 1$ and IL4R, which showed an upward trend, while the genes that promote the immune function, such as IL-15, $-16,-17 R A,-1 A$, and $-1 R N$, initially showed an upward trend, following by a downward trend (Fig. 4E). Correlation with the tissue origins showed that, once monocytes from the blood entered the tumor tissues, they differentiated into M1-like macrophages and gained increased immune function and M2-like TAMs, alongside enhanced immunosuppressive effects.

SCENIC analysis was performed to determine the changes in TFs during the transformation of macrophages into TAMs (Fig. 4F, G). We found that MAF, ETV5, and EGR2 M2-like transcription factors were highly expressed in TAMs associated with immunosuppression. MAF regulates the activation of the Treg and IL-4 pathways ${ }^{67}$. ETV5 is related to blood vessel growth and activation of the IL-10 pathway ${ }^{8,33}$. The expression of EGR2 in CD4 + T cells is related to the conversion of CD4 + T into Tregs and the activation of the IL-4 and TGF- $\beta$ functional pathways ${ }^{42,63}$. Conversely, STAT4, NFKB1, NFKB2, RUNX1, and BCL3 M1-like transcription factors are enriched in macrophages. STAT4 mediates the JAK-STATrelated pathways and participates in the conduction of the IL-12, $-21,-23$, and -35 signaling pathways ${ }^{38}$. NFKB1 and NFKB2 promote the polarization of macrophages to the M1 type ${ }^{16}$. BCL3 acts synergistically with NF-KB ${ }^{61}$. 
Finally, we utilized CellChat to investigate interactions between TAMs and cell subgroups in the TME (Fig. 5D). Interestingly, compared to macrophages, TAMs participated more in the construction of the immunosuppressive microenvironment. The immunosuppressive ligands secreted by TAMs, such as CD80, CD86, CD274, ICOSL, and NECTIN2, showed evident interactions with other receptors such as CTLA-4, PD-1, ICOS, and TIGIT expressed by other cells, especially T cells (Fig. 5E, F, G). Network analysis showed that TAMs were the main senders of CD80, CD86, and ICOSL, whereas Tregs, as vital components of active intertumoral immunosuppression, were the main receivers. In addition to IRS, TAMs participating in the construction of the immunosuppressive microenvironment secreted soluble cytokines such as IL-10 and SPP1 (Fig. 5A). Interestingly, TAMS were both the main secretors and receivers of IL-10, whereas macrophages were the main receivers of IL-10 (Fig. 5B). This suggested a positive feedback loop between macrophages and TAMs. Once macrophages had differentiated into TAMs, TAMs possibly secrete IL-10 acting on macrophages to promote the differentiation process. TAMs also secreted SPP1, which interacted with almost all cells in the TME, including DCs, Tregs, Tex, fibroblasts, and malignant cells (Fig. 5C). Interestingly, TAMs were also the main receivers. SPP1 mediates macrophage polarization and facilitates immune escape in lung adenocarcinoma ${ }^{75}$. It may be attributable to the M2 phenotype of TAMs in this study.

\subsection{Lipid metabolism reprogramming in TAMs}

The DEGs calculated between macrophages and TAMs suggested the involvement of genes associated with lipid metabolism because Peroxisome Proliferator Activated Receptor Alpha (PPARA) was highly expressed in TAMs. Enrichment analysis revealed that, compared with macrophages, TAMs were more enriched in lipid metabolism pathways, such as the cholesterol biosynthesis pathway, cholesterol metabolism, fatty acid metabolism, and mitochondrial fatty acid beta-oxidation (Fig. 4B). GSVA analysis also revealed that the cholesterol, lipid droplet synthesis, and lipid catabolic pathways were enhanced in TAMs (Fig. 4C). Pseudotime analysis showed that the expression of genes associated with lipid output such as $A B C A 1$ and $A B C G 1$ were enhanced in the differentiation process (Fig. $4 \mathrm{E}$ ). It is reasonable to speculate that, along with differentiation, metabolism patterns were remodeled toward lipid metabolism.

Pathways such as the PPAR alpha pathway, regulation of cholesterol biosynthesis by sterol regulatoryelement binding proteins (SREBP: SREBF), NR1H2 and NR1H3 mediated signaling, and NR1H3 and $\mathrm{NR} 1 \mathrm{H} 2$ regulated gene expression linked to cholesterol transport and efflux, were also enriched in TAMs (Fig. 4F, G). SREBP functions in the transcriptional regulation of genes involved in the biosynthesis and uptake of lipids, promoting fatty acid synthesis and inducing TAMs instead of M2 ${ }^{37,55}$. NR1 H2 and $\mathrm{NR} 1 \mathrm{H} 3$ act as transcription factors engaged in lipid metabolism synthesis and are important modulators of the SREBP-1c pathway at the transcription level, where they regulate gene expression linked to cholesterol transport and efflux in hepatic lipogenic cells ${ }^{19}$. We were particularly interested in the cholesterol efflux function mediated by NR1H2 and NR1H3. Increased cholesterol outflow increased lipid content in the TME to provide nutrition for tumor cell growth and destroyed the lipid raft of TAMs to weaken the TLR4 signaling pathway ${ }^{19}$. Increased cholesterol outflow also enhanced the IL-4 pathway, weakened the interferon (IFN) pathway ${ }^{22}$, and has an unexpected role in the polarization of TAMs to M2. 
We speculated that reprogramming of lipid metabolism in TAMs is involved in the remodeling of immune functions, to a certain extent.

SCENIC analysis reported the abnormal transcriptional regulatory network in TAMs. As shown in Fig. 4G, M1 transcription factors are significantly activated in TAMs, which mainly include the SREBF and NR1H3 transcription factors. Therefore, SREBF and NR1H3 may play important roles in lipid metabolism reprogramming in TAMs. TAMs and Tex have both undergone lipid metabolism remodeling, reflecting the important role of lipid metabolism in the process of T cell exhaustion and TAMs polarization to M2. However, there are significant differences between these two kinds of cells, which are mainly manifested in the differences in the transcription regulatory factors. Hence, we suspect that SREBF and NR1H3 may be important targets to prevent or reverse the polarization from TAMs to M2.

\subsection{DCs exhibit a similar pattern to TAMs in metabolism remodeling and construction of the immunosuppressive microenvironment}

DCs are the most potent antigen-presenting cells in the immune system and are central players in the adaptive immune response. The myeloid cell sub-clusters 5 and 7 were annotated as DCs. However, as cluster 7 only contained 18 cells, cluster 5 was of more interest. Marker gene analysis revealed that DCs in cluster 5 exhibited highly expressed immunosuppressive cytokines, such as IL-4, -10 , and IFNa/ $\beta$. Enrichment analysis revealed that IL-10, -4 , and -13 signaling, interferon alpha/beta signaling, PD-1 signaling, and CTLA-4 inhibitory signaling were enriched. CellChat analysis found DCs exhibited a similar pattern to TAMs in secreting immunosuppressive cytokines, especially CD80, CD86, and ICOSL (Fig. 4B). Network analysis demonstrated that DCs and TAMs were the main secretors participating in the exhaustion process of CD8 T cells, synergistically promoting the construction of the immunosuppressive microenvironment.

At the same time, enrichment analysis revealed that lipid metabolism, fatty acid metabolism, and PPARAactivated gene expression pathways were highly enriched in DCs. PPARs control the expression of many genes involved in metabolic homeostasis, and lipid and glucose metabolism. Except for aberrant lipid storage, the PPARs pathway also controls glucose metabolism. Thus, the tricarboxylic acid(TCA) cycle was enhanced, resulting in citric acid accumulation. These conditions provide the substrate for the de novo synthesis of fatty acids and intracellular lipid droplets. Other pathways were also enriched, including Wnt signaling and CDK-beta-catenin activity. Wnt5 can act on Frizzled (FZD) family receptors on DCs and trigger the activation of downstream PPAR pathways through activation of $\beta$-catenin signals to remodel lipid metabolism in melanoma ${ }^{52}$. Other pathways enriched in DCs included RUNX3, which regulates Wnt signaling, transcriptional regulation by RUNX2 and regulation of RUNX2 expression and activity (Supplementary Fig. 3D). SCENIC analysis demonstrated that RUNX2 was highly expressed in DCs in cluster 5 . These data implied that lipid metabolism remodeling in DCs might also depend on the core Wnt/ $\beta$-catenin/PPAR signaling pathway regulated by the RUNX family. 
Lipid metabolism reprogramming mainly comprised lipid storage but also included enhanced lipid oxidation metabolism in DCs. Regarding the TAMs, we speculate that lipid metabolism reprogramming in DCs is involved in reconstructing the immunosuppressive microenvironment.

\section{Fibroblast cells}

We extracted 145 fibroblast cells classified into two clusters: fibroblast-1 and fibroblast-2 (Fig. 6A). Pseudotime analysis revealed that fibroblast- 1 was present at the initial stage of the differentiation trajectory, and fibroblast-2 was present at the end (Fig. 6E, F).

\subsection{Hypoxia and acid signals promote metabolic and functional remodeling in fibroblasts}

Interestingly, DEGs enrichment analysis revealed different metabolic patterns between the two clusters (Fig. 6B). The pathways related to lipid metabolism, such as the cholesterol metabolism pathway, metabolism of lipids, and fatty acid metabolism, were significantly enriched in fibroblast-2. In contrast, pathways related to carbohydrate metabolism, such as glycogen metabolism, and the glycolysis and gluconeogenesis pathways were enriched in fibroblast-1 (Fig. 6C). GSVA analysis revealed the differences in metabolic patterns between the two sub-clusters (Fig. 6D). Cells in fibroblast- 1 obtained their energy predominantly through the glycolytic pathway, whereas those in fibroblast-2 obtained their energy through the lipid metabolism pathway. We focused on several enrichment pathways highly enriched in fibroblast-2, such as RUNX2, NR1H3, and NR1H2, which regulate gene expression linked to cholesterol transport and efflux, and NR1H2 and NR1H3-mediated signaling. SCENIC analysis also showed that lipid genes regulated by NR1H2, NR1 H3, and RUNX were upregulated in fibroblast-2 (Supplementary Fig. 3D). Our analyses revealed that enhanced lipid metabolism and abnormal lipid accumulation may occur in Tex, TAMs, and fibroblast-2.

Further GNA analysis of aberrant lipid metabolism in fibroblast-2 and - 1 disclosed that G-protein-coupled receptors (GPCRs) pathways were highly expressed in fibroblast-1. GPCRs include seven transmembrane domain proteins that relay numerous extracellular signals and react, especially, to acid and hypoxia signals ${ }^{29}$. It was previously shown that hypoxia could induce macrophages to differentiate into TAMs in the tumor microenvironment and promote cancer progression ${ }^{48}$. Hypoxia-mediated acidification blocks the capacity of T cells to expand or perform cytotoxic effector functions, thus causing immunosuppression ${ }^{60}$. Hypoxia and acid signals have prominent roles in establishing and maintaining tumor immunosuppression. Aberrant signals in the TME via GPCRs receptors may cause abnormal lipid metabolism. Interestingly, SCENIC analysis also found that hypoxic stress signals such as HIF-1A, EPAS1 (HIF-2A), and ETS1 transcription factors were present at high levels in fibroblast-1 (Supplementary Fig. 3D). We speculate that hypoxia is an effective stimulus that induces fibroblast differentiation.

\subsection{The positive feedback loop of Wnt and BMP in fibroblasts mediating metabolic remodeling}


Functional enrichment analysis and GSVA showed highly enriched Wnt and bone morphogenetic protein (BMP) pathways in fibroblast-2 representing the final stage of the differentiation trajectory (Fig. 6D). Previous studies proved that Wnt ligands bind to the transmembrane FZD receptor in the canonical Wnt pathway, activating Disheveled (Dvl), and then triggering the stabilization and accumulation of nuclear $\beta$ catenin transcriptional activity. $\beta$-catenin significantly modulates de novo lipogenesis through the PPAR- $\gamma$ pathway in breast cancer ${ }^{64}$ and hepatocellular cancer ${ }^{6}$. We speculate that over-activation of Wnt signaling mediates $\beta$-catenin accumulation, which further activates PPAR- $y$ signaling and may be attributed to aberrant lipid metabolism in fibroblast-2. Like Tex and TAMs, we speculate that lipid metabolism reprogramming in fibroblast-2 may be involved in constructing immunosuppressive environments like TAMs.

We utilized CellChat to investigate the source of the Wnt ligands. Unexpectedly, the major secretors of Wnt ligands were fibroblast cells rather than receptors in the TME (Fig. 6H). More importantly, compared to fibroblast-1, fibroblast-2 secreted more Wnt ligands, such as Wnt2B, Wnt4, and Wnt5A, which interacted with receptors expressed on malignant and endothelial cells (Fig. $6 \mathrm{~J}$ ). Interestingly, fibroblast-2 secreted more BMPs, such as BMP2, $-4,-5$, and $-8 \mathrm{~B}$, which could act on themselves through the autocrine or paracrine pathways (Fig. 6I). Network analysis of the BMP pathways revealed that fibroblast-2 was the major sender of BMP ligands, whereas the main receivers were fibroblast-1, endothelial cells, and malignant cells. Activation of BMP signaling inhibited the expression of $\beta$-Trcp, increasing the nonphosphorylated form of $\beta$-catenin in the classic Wnt signaling pathway and promoting lipids synthesis ${ }^{73}$. These results implied that over-activation of the Wnt- $\beta$-catenin-PPAR-y pathway in fibroblast cells depends on BMP signals secreted by themselves to a large extent. We could reasonably speculate the presence of two positive feedback loops in fibroblast cells. First, the GPCRs expressed in fibroblast- 1 act as a result of the hypoxia and acid signals caused by tumor cells initiating differentiation into fibroblast2. Then, fibroblast-2 secretes more Wnt ligands to act on tumor cells to promote the malignancy phenotype. Second, fibroblast-2 secretes BMPs acting on fibroblast- 1 to promote its differentiation and metabolism remodeling by enhancing the Wnt- $\beta$-catenin-PPAR-y pathway.

The above results demonstrate that fibroblast-2 engages more in reconstructing the TME to promote tumor progression than fibroblast-1. However, knowing the underlying mechanism provides new targets to reverse the differentiation of fibroblast cells by blocking hypoxia and acid signaling, GPCR receptors, BMPs, and Wnt ligands, for example. Similar to Tex and TAMs, the similar transcription pattern strongly illustrates the potential of these transcription factors to act as targets to reverse the metabolic remodeling of cells in the TME.

\section{Malignant cells}

We identified nine sub-clusters in epithelial cells, which were divided into five cell types according to the CellMarker database and Zhang Lei et al $^{72}$ : malignant cells, goblet cells, enterocytes, stem-like cells, and undefined cells (Fig. 7A). When the origin of the epithelial cells was traced, it was noted that malignant cells and stem-like cells occurred almost exclusively in tumor tissues, while enterocytes and goblet cells 
occurred in tumor tissues and normal tissues. Since CRC cells originate from enterocytes, we focused on the differences between malignant cells and enterocytes.

\subsection{Abnormal metabolism of malignant cells}

We performed enrichment analysis of the DEGs between malignant cells and enterocytes (Fig. 7B). As shown in Fig. 7C, glycolysis, lipid biosynthesis, and amino acid metabolism pathways were enriched in cancer cells, while oxidative phosphorylation was suppressed. GSVA analysis also disclosed that PPAR, adipogenesis, and lipid metabolism pathways were enriched in malignant cells, consistent with previous studies (Fig. 7D).

SCENIC analysis showed enhanced HIF-1a in malignant cells (Supplementary Fig. 4A). HIF-1a is associated with glucose metabolism, which shifts glucose metabolism from highly efficient oxidative phosphorylation to less efficient glycolysis by regulating downstream transcription factors and providing substrates for lipid accumulation ${ }^{49}$. Interestingly, NPAS2 and ATF4 were also highly enhanced in malignant cells. NPAS2 is a core circadian molecule that binds to the E-box site in the HIF-1 a promoter region and thus promotes HIF-1a mRNA expression in hepatocellular cancer ${ }^{69}$. ATF4 is a direct transcription target of HIF-1a and is involved in amino acid metabolism, such as the biosynthesis of asparagine, serine, and glycine $24,50,54$. We speculate that NPAS2 regulates HIF-1a, and that they jointly mediate downstream metabolism processes. NPAS2 and ATF4 may be potential therapeutic targets in CRC.

Enrichment analysis enabled the classification of all the TFs to different modules and showed that the aryl hydrocarbon receptor (AhR) was enriched in Module 2. The AhR is activated by tryptophan catabolites, enhances tumor malignancy, and suppresses anti-tumor immunity in glioblastoma ${ }^{51}$. Changes in intracellular and extracellular metabolites have profound effects on cancer progression by enhancing the ability of cancer cells to survive and proliferate.

\subsection{The Wnt pathway is linked to cancer progression}

As shown in Fig. 7C, variance and GSVA analyses revealed that the Wnt and Hedgehog pathways were significantly enhanced in colorectal malignant cells. This enhancement is critical for cancer initiation and progression 7,10 .

Subsequently, CellChat analysis revealed that the activation of the Wnt pathway in malignant cells contributes to the presence of many cell types in the TME. Tex, fibroblast-1, and TAM DCs jointly secrete Wnt2B and Wnt4, which act on FZD and LRP receptors expressed on malignant cells. This initiates the $\beta$ catenin-dependent pathway and promotes the progression of cancer (Fig. 7E). In addition, malignant cells can directly secrete BMP2 and - 4 molecules, which interact with their own BMPR1 and BMPR2 receptors, enhancing the expression of Wnt3 and Wnt3a and indirectly regulating the classical Wnt pathway.

BMPR1 or BMPR2 have been reported to be mutated in over $70 \%$ of cases of $\mathrm{CRC}^{23}$. There is increasing evidence in sporadic colon cancers that mutations affecting BMP signaling corroborate with activated 
Wnt to drive colon cancers, particularly in the later stages ${ }^{26}$, thus providing a rationale for exploiting these pathways as potential targets in the treatment of colon cancer.

Accumulating evidence has shown that activation of Wnt promotes the accumulation of $\beta$-catenin ${ }^{53}$. However, enrichment analysis also found $\beta$-catenin independently of the Wnt pathway, demonstrating that other molecules are involved in the biosynthesis of $\beta$-catenin. SCENIC analysis reported the upregulation of SOX9 and XBP1 transcriptional factors in malignant cells (Supplementary Fig. 4C). SOX9 participates in regulating the downstream Wnt pathway by combining directly with $\beta$-catenin ${ }^{18}$. SOX9 can also combine with $\mathrm{GLI}$, downstream molecules of the hedgehog pathways, thus maintaining the stem phenotype of malignant cells. XBP1 can promote the accumulation of $\beta$-catenin in endothelial cells via the Akt/GSK pathway ${ }^{71}$. We speculate that Wnt, SOX9, and XBP1 jointly regulate the activation of downstream $\beta$-catenin, inducing a series of malignant functions and the remodeling of metabolism, such as lipid metabolism via the PPAR pathway.

We also enriched TFs related to the epithelial-mesenchymal transition (EMT) pathway, such as ONECUT2 and ETV4 in colorectal malignant cells (Supplementary Fig. 4D). ONECUT2 is involved in the EMT pathway, migration, and invasion of CRC cells ${ }^{57}$. In breast cancer, ETV4 targets MMP13, an extracellular metalloprotease, promoting tumor metastasis ${ }^{17}$. In lung cancer, ETV4 regulates PXN and $M M P 1^{65}$. In gastric cancer, ETV4 upregulates the expression of KIF2A via the AKT pathway, promoting tumor migration ${ }^{74}$. Interestingly, SOX9 and XBP1 also engaged in the EMT pathway ${ }^{35,36}$, and these TFs are potentially new therapeutic targets.

\subsection{CRC cells engaged in constructing the immune- suppressive microenvironment}

Inhibitory signals mediated by malignant cells responsible for constructing the immune-suppressive microenvironment are classified into two major categories: immunosuppressive pathways and soluble molecules secreted by malignant cells.

Variance analysis showed that TGF- $\beta$ pathways were highly activated in malignant cells, including signaling by TGFB family members, the TGF-beta signaling pathway, TGF-beta receptor signaling, which activates SMADs, and TGF-beta receptor signaling in the EMT pathways (Fig. 7H). GSVA analysis also significantly enriched the TGF- $\beta$ pathways. TGF- $\beta$ possesses dual tumor-suppressive and tumorpromoting roles in tumor progression ${ }^{14}$. Tumor-derived TGF- $\beta$ can induce tumorigenic and pro-metastatic responses in cancer cells, including the formation of an immune-suppressive $\mathrm{TME}^{5}$.

CellChat analysis showed that NECTIN2 and ICOSL ligands have obvious interaction with immunosuppressive receptors expressed by Tex, such as TIGIT, CTLA-4, and ICOS (Fig. 7E). Our results implied that malignant cells might speed up the exhaustion process of Teff, thus promoting the construction of the immunosuppressive microenvironment. We found that malignant cells mainly secrete inflammatory factors that induce the production of immunosuppressive cells, such as Tex, DCs, and 
TAMs (Fig. 7F). For example, SPP1 molecules secreted by malignant cells mediate macrophage polarization and facilitate immune escape in lung adenocarcinomas ${ }^{75}$. Together, these molecules form a network to create an unfavorable immunosuppressive microenvironment.

\section{The infiltration of tumor-educated immune cells is associated with a worse prognosis in CRC}

We performed digital cytometry analyses using CIBERSORTx to evaluate the abundance of tumor stromal and immune cell subsets analyzed previously in patients from The Cancer Genome Atlas-Colon Adenocarcinoma (TCGA-COAD) data. We established a new risk model using stepwise regression to evaluate the association between cell fractions and prognostic outcomes and identify the optimal coefficient for each population. Finally, we selected eight subgroups to construct the model. The formula for the risk model is as follows:

Riskscore $=4.785 *$ Fibroblast_cells. $1+4.222 *$ Myeloid.cell.5 $-2577.080 *$ Myeloid.cell. 2 $86.019 *$ Myeloid.cell.3 + 10.550*CD8_T_cells.2 + 4.868*CD8_T_cells.4 + 9.276*CD8_T_cells.0$13.466 *$ CD8_T_cells.5.

$$
\text { Riskscore }=\sum_{i=1}^{n} \text { Coef }_{i} * \text { Fraction }_{i}
$$

The patients were divided into high-risk and low-risk groups according to the median risk score. Next, we evaluated the prognostic value of the risk model for overall survival (OS). Patients in the high-risk group had a significantly worse OS than the low-risk group $(p=0.011)$ (Fig. $8 \mathrm{C})$. The model's accuracy was verified using time-dependent receiver operating characteristic (ROC) curves, which confirmed the reliability of the prognoses for both cohorts. The area under the ROC curve for the risk score was 0.814 , 0.678 , and 0.640 for $1-, 3$ - and 5 -year OS versus $0.792,0.663$, and 0.660 for TNM stage, respectively (Fig. $8 \mathrm{E})$. These scores show that the risk model possesses the clinical predictive value supplementing the TNM stage to some extent. Furthermore, we used a multivariate Cox regression model to construct a new clinical model incorporating risktype, TNM stage, gender, and age (Fig. 8A). Interestingly, risktype, TNM stage, and age were independent prognosis factors $(p=0.031,0.020$, and 0.006 , respectively) (Fig. 8B). The patients were separated into two subgroups according to the median clinical risk score. Kaplan-Meier (KM) survival analysis revealed that high clinical risktype had a significantly worse OS than low clinical risktype (Fig. 8D). The areas under the ROC curve for 1-, 3-, and 5-year OS were $0.870,0.754$, and 0.767 , respectively, which was better than the risk model and TNM stage (Fig. 8F).

\section{Materials And Methods}

\section{Data acquisition}


The scRNA-seq data and bulk RNA-seq data of human CRC samples were included in this study. The scRNA-seq profiles included 10,398 cells from 10 human CRC samples (accession number GSE146771), which were obtained from the Gene Expression Omnibus (GEO, http://www.ncbi.nlm.nih.gov/geo/) database. This database contains 5169 cells from tumor cores, 2400 cells from paracancerous tissues, and 2829 cells from peripheral blood, performed using the SMART-seq2 platform. Normalized matrix files for the dataset were downloaded. The bulk RNA-seq data of CRC samples, including 398 tumor samples and 39 normal samples, were obtained from the TCGA database (https://portal.gdc.cancer.gov/).

We excluded samples with an overall survival (OS) time $<7$ days or insufficient clinical information regarding age, gender, or TNM stage.

\section{Processing of the CRC scRNA-seq data and bulk RNA-seq data}

A total of 10 samples and 10,398 cells were included in this study. The Seurat package in R 4.0.3 was used for quality control (QC). The quality standards were as follows: 1 ) genes detected in $<3$ cells were excluded; 2 ) cells with $<50$ total detected genes were excluded, and; 3 ) cells with $\geq 5 \%$ of mitochondriaexpressed genes were excluded. For the remaining cells, cell-cycle scores were calculated using Seurat's CellCycleScoring function since the cell cycle phase effect was observed. Batch effects among the patients had already been eliminated by the data donator. The gene expression matrices were further normalized to RNA counts, mitochondrial percentages, and cell cycle scores using the top 3000 variable genes. PCA was used to calculate the significantly available principal components (PCs). We then applied the t-distributed stochastic neighbor-embedding (tSNE) algorithm for dimensionality reduction with 20 initial PCs to perform cluster classification analyses across all cells.

\section{Cell type recognition}

We performed differential expression analysis among all genes within cell clusters using Seurat's FindAllMarkers function to identify the marker genes in each cluster. An adjusted P-value $<0.05$, expression percentage $>0.25$, and | log2 [fold change $(F C)$ ] $\mid>0.25$ were considered cutoff criteria for identifying marker genes. Subsequently, different cell clusters were determined and annotated by the singleR package according to the composition patterns of the marker genes and were then manually verified and corrected with the CellMarker database. The malignant cells were annotated by correlation with the data donator's cell annotation of malignant cells. The corresponding genes of cell surface markers for the annotation of cell clusters are listed in Supplementary Table 1.

\section{Pseudotime trajectory analysis}

Single-cell pseudotime trajectories were constructed using the Monocle 2 algorithm, an R package designed for single-cell trajectories by Qiu et al. to reveal the immune cell differentiation process. This algorithm applies a machine learning technique to reduce the given high-dimensional expression profiles to a low-dimensional space, visualized as a tSNE plot. Single cells were projected onto this space and ordered into a trajectory with branch points. The dynamic expression heatmaps were constructed using 
the plot pseudotime heatmap function. In addition, differential expression analysis between branches was performed using the plot_genes_branched_heatmap function.

\section{Functional enrichment analysis}

DEGs analysis was performed using Seurat's FindMarkers function. The following cutoff threshold values were used: adjusted P-value $<0.05$ and $|\log 2[\mathrm{FC}]|>1$. The DEGs were loaded into Metascape (http://metascape.org), a tool for gene annotation and gene list enrichment analysis.

GSVA was performed to explore correlating pathways of different cell clusters. We downloaded "c2.all.v7.4.symbols.gmt" and "c5.all.v7.4.symbols.gmt" from the Gene Set Enrichment Analysis website (http://www.gsea-msigdb.org), which were used for further enrichment analyses. The GSVA analysis was performed in R 4.0.3 to calculate the enrichment score of the pathways in each cell.

\section{Cell-cell communication analysis}

CellChat is a versatile toolkit used to infer and analyze intercellular communication networks from scRNA-seq data quantitatively. Based on the ligand-receptor interactions database for humans and mice and using network analysis and pattern recognition approaches, CellChat can predict major signaling inputs and outputs for cells and establish how those cells and signals coordinate their functions. As described above, 10,398 single cells were clustered into 28 cell types. CellChat was then applied to analyze the molecular interaction among the 28 cell types. Ligand-receptor pairs with a P-value $<0.05$ were filtered to evaluate the relationship between different cell types.

\section{Gene regulatory network analysis}

We used SCENIC (Aibar et al., 2017), an algorithm package that can reconstruct transcriptional states and regulatory networks from scRNA-seq data, to access the gene regulatory networks relating to TFs and regulons in individual cells of the $\mathrm{CRC}$ samples. The gene expression matrix of each cell type was input into SCENIC, and a co-expression network was constructed using GENIE3. Direct binding by DNA-motif analysis was identified based on a motif dataset (hg19-500bp-upstream-7species.mc9nr.feather, hg19tss-centered-10kb-7species.mc9nr.feather) to construct regulons for each transcription factor. Finally, regulon activity was analyzed using AUCell (Area under the Curve), where a default threshold was applied to binarize the specific regulons ("0" = "off" for TFs, and "1" = "on"). Regulon modules were identified based on the Connection Specificity Index (CSI) to identify specific associating partners. Hierarchical clustering with Euclidean distance was performed based on the CSI matrix to identify different regulon modules. We then used 0.65 as a cutoff to construct the regulon association network, to investigate the relationship between different regulons.

\section{Correlation with bulk RNA-seq data}

CIBERSORTX is a new machine learning method developed from CIBERSORT for estimating the abundance of cell clusters in bulk RNA-seq data. This tool was used to digitally purify the transcriptome 
of individual cell clusters from the bulk data without isolating single cells. Based on the scRNA-seq data identified previously, we applied CIBERSORTx to create a signature matrix. Next, we used CIBERSORTx to estimate the fraction of each cell cluster in TCGA CRC expression matrix, which was first normalized to transcripts per million (TPM) values. Next, multivariate Cox regression and stepwise regression analyses were applied to select the optimal coefficient for each cell cluster to construct the risk model. The risk scores were then divided into "high risktype" and "low risktype" according to the median risk score. The formula for the model is as follows:

$$
\text { Riskscore }=\sum_{i=1}^{n} \text { Coef }_{i} * \text { Fraction }_{i}
$$

Then we incorporated the risktype, TNM stage, gender, and age to construct a new clinical risk model using multivariate Cox regression. The clinical risk scores were divided into "high clinical risktype" and "low clinical risktype" according to the median clinical risk score. The formula for the clinical model is as follows:

Clinical Riskscore $=\sum_{i=1}^{n}$ Coef $_{i} *$ factor $_{i}$

The associations of risktype and clinical risktype with OS were analyzed using KM curves with ROC curve analysis to verify the sensitivity and specificity of the model for the training cohort.

\section{Statistical analyses}

Statistical analyses were conducted using R software (version 4.0.3; R Foundation for Statistical Computing, Vienna, Austria). All statistical tests were two-sided, with P-values $<0.05$ considered statistically significant.

\section{Conclusion}

Treatment of CRC, especially advanced CRC, remains challenging. Despite developments in treatments for CRC such as immunotherapy and targeted therapy, only a small number of people benefit from these treatments, and this low number is attributed to low efficiency and high drug resistance. A recent study found that cancer cells and tumor-infiltrating cells take part in the process of drug resistance or fail to respond to cancer treatment strategies. The solution lies in a deeper understanding of the TME. This study investigated the TME of CRC using scRNA-seq and revealed the heterogeneity of the immune cells and stromal cells within CRC tissues. We identified various cell types and altered pathways, along with intercellular communication networks and novel regulatory networks driven by TFs in CRC. On this basis, we identified the convergence in different cell types by investigating the various differentiation stages of 
each cell type. Our results will aid our understanding of intertumoral heterogeneity and homoplasy in CRC and provide potential targets for cancer therapy.

To describe the TME fully in CRC is not possible. We selected certain cell types, such as CD8 + T cells, myeloid cells, fibroblast cells, and epithelial cells, representing the main components of the TME, to illustrate its heterogeneity and convergence. Our key conclusions are as follows:

First, we identified that the immunosuppressive microenvironment in CRC consisted of immune cells and stromal cells. The differentiation trajectory of each cell type revealed that the cells closer to the end of their trajectory showed more immunosuppressive characteristics, such as exhaustion in CD8 $+\mathrm{T}$ cells and polarization to the M2 phenotype in macrophages and fibroblast-2. In this process, proinflammatory functions were inhibited, whereas immunosuppression functions were enhanced. In addition, the intercellular communication network showed more active secretion of immunosuppressive cytokines by cells closer to the end of their differentiation trajectory.

For example, in the regulation of IRS, exhaustion was the inevitable outcome of CD8 + T cells mediated by various cells in the TME. At the same time, different cells manifested different ligand modes. Tumor cells mainly secreted PVR and NECTIN2 to act on the TIGIT receptor. CD80 and CD86 secreted by DCs and TAMs interacted with CTLA and Tregs and mainly secreted CD274 to act on PDCD1. Network analysis also showed that IRS were expressed in Tex and immunosuppressive cells such as TAMs, Tregs, and DCs. Soluble cytokines such as IL-10 and SPP1 were also secreted by TAMs that participated in constructing the immunosuppressive microenvironment. Interestingly, the network analysis of IL-10 implied the potential of a positive feedback loop between macrophages and TAMs to promote the differentiation process. SPP1 was mainly secreted by TAMs, whereas the main receivers were fibroblast cells and TAMs. The positive feedback loop may equally be applied to SPP1 in TAMs to maintain the M2 phenotype.

As mentioned above, we mapped the differentiation pathways of each subgroup of cells and found that different clusters of each cell type had different metabolic patterns. We identified the DEGs of the two most representative cells in each cell subgroup for functional enrichment analysis to demonstrate the differences more intuitively. This included Teff and Tex, M1-like macrophages and M2-like TAMs, fibroblast- 1 and fibroblast-2, and malignant cells and enterocytes. Interestingly, although the metabolic patterns were diverse, those cells close to their terminal differentiation showed similar metabolic patterns, i.e., enhanced lipid metabolism, manifesting as the abnormal accumulation of lipids.

SCENIC analysis revealed that the transcription factors that regulate lipid metabolism remodeling in each subgroup partially overlapped. The most representative transcription factors are PPARG, SREBF, NR1H2, and NR1 H3. In connection with the changes in immune functions, the transformation in the metabolic model was highly consistent with the transformation of the immune functions. The NRIH2 and NR1H3 transcription factors were enriched in M2-like TAM and were of particular interest. The genes regulated by $\mathrm{NR} 1 \mathrm{H} 2$ and NR1H3 were linked to cholesterol transport and efflux, and the outflow of cholesterol could destroy the lipid rafts on cell membranes, attenuating the TLR4 signaling pathway. Furthermore, increased cholesterol outflow also enhanced the IL-4 pathway and attenuated the IFN pathway. This 
phenomenon implied that enhanced intracellular lipid metabolism might be an important factor in the transformation of immune function, and transcription factors involved in lipid metabolism remodeling in cells may be potential therapeutic targets to reverse immunosuppression.

We applied CIBERSORTx in our study of the TME in CRC to investigate the link between the fraction of cell subgroups and prognosis. Our risk model incorporated eight cell subgroups, including Fibroblast_cells.1, Myeloid.cells.5, Myeloid.cells.3 and CD8_T_cells.5, which are associated with oncogenesis and progression in tumors, according to previous scRNA-sequencing analyses. Compared to the classical TNM stage, based on the ROC curve analysis, our risk model was proven to be useful as a novel prognostic tool for improving survival prediction accuracy in patients with colon cancer. To investigate whether the risk model could supplement the TNM stage in survival prediction, we established a new clinical risk model, including risktype, TNM stage, gender, and age. The AUC for 1-, 3-, and 5-years was significantly better than either the risk model or TNM stage. We showed that this risk model, based on cell fractions in the TME, could supplement the gold standard in clinical practice and represents an alternative method that shows promise in clinical practice applications.

More importantly, with advances in sequencing technology, the cost of normal bulk RNA-sequencing will decrease while the accuracy of scRNA-sequencing will improve. This means that more patients with colon cancer will be able to afford the cost of bulk RNA-sequencing while benefitting from the increased accuracy of scRNA-sequencing using CIBERSORTx. This type of deconvolution algorithm could deconstruct bulk RNA-sequencing data and apply scRNA-sequencing data to reveal previously ignored information. Our results provide opportunities to improve the prognostic standards currently in use.

In summary, this study provides a new perspective for understanding the heterogeneity and homoplasy of the TME and will aid the development of immunotherapies to treat CRC.

\section{Abbreviation}

CRC colorectal cancer

SCENIC single-cell regulatory network inference and clustering

TCGA The Cancer Genome Atlas

HER2 human epidermal growth factor receptor 2

EGFR epidermal growth factor receptor

TME tumor microenvironment

Tex exhausted CD8 T cell

TAM tumor-associated macrophages 
CAF cancer-associated fibroblasts

ICB Immune checkpoint blockade

PD-1 programmed cell death 1

CTLA-4 cytotoxic T lymphocyte-associated protein 4

dMMR mismatch-repair-deficient

MSI-H microsatellite instability-high

MSI microsatellite instability

IRS inhibitory receptors

scRNA-seq single-cell RNA sequencing

TNM the tumor, nodes, and metastasis

QC quality control

ILC innate lymphoid cell

DC dendritic cells

BEAM branch expression analysis modeling

Teff T effector cell

DEG differentially expressed genes

AMPK Adenosine 5'-monophosphate -activated protein kinase

GSVA gene set variation analysis

FA fatty acid

SREBP Sterol regulatory element binding protein

FASN atty acid synthase

ACC Acetyl-CoA Carboxylase

HMG-CoA 3-hydroxy-3methylglutary-coenzyme A

ACLY ATP-citrate lyase 
PPAR peroxisome proliferator-activated receptor

TF transcription factors

TGF- $\beta$ transforming growth factor-beta

$\mathrm{IFNa} / \beta$ interferons alpha and beta

TNF tumor necrosis factor

IL interleukin

Treg regulatory $\mathrm{T}$ cells

PVR poliovirus receptor

TIGIT T cell immunoreceptor with immunoglobulin and ITIM domain

ICOSL/ICOS Inducible Co-Stimulator Ligand/ Inducible Co-Stimulator

TIM3 the inhibitory receptor T-cell immunoglobulin and mucin domain 3

LAG3 lymphocyte activation gene 3 protein

MSI-L microsatellite instability-low

VEGF vascular endothelial-derived growth factor

ZEB1 zinc finger E-box binding homeobox 1

MMP matrix metalloproteinase

TLR4 Toll-like Receptor 4

TCA tricarboxylic acid

FZD Frizzled

GPCR G-protein-coupled receptor

BMP bone morphogenetic protein

Dvl Disheveled

AhR aryl hydrocarbon receptor

EMT epithelial-mesenchymal transition 
TCGA-COAD The Cancer Genome Atlas-Colon Adenocarcinoma

OS overall survival

$\mathrm{ROC}$ receiver operating characteristic

KM Kaplan-Meier

GEO Gene Expression Omnibus

PC principal component

tSNE t-distributed stochastic neighbor-embedding

CSI Connection Specificity Index

TPM transcripts per million

NR1H3 Liver $X$ receptors alpha

NR1H2 Liver $\mathrm{X}$ receptors beta

\section{Declarations}

\section{Ethics approval and consent to participate}

Not applicable.

\section{Consent for publication}

The authors confirm that the work has not been published before nor elsewhere.

\section{Availability of data and materials}

The scRNA-seq profiles included 10,398 cells from 10 human CRC samples (accession number GSE146771), which were obtained from the Gene Expression Omnibus (GEO, http://www.ncbi.nlm.nih.gov/geo/) database.

\section{Competing interests}

The authors declare that they have no competing interests.

\section{Funding}

This work was supported by grants from the National Natural Science Foundation of China (No. 81773065); Natural Science Foundation of Zhejiang Province (No. LY21H160023). 


\section{Authors' contributions}

YJ and MJS designed the study and reviewed the literature. XSY performed the data analyses and wrote the manuscript for the study. CYK and CDL downloaded the data from GEO database and participated in the drafting of the manuscript. CW and YB contributed to the reviewing of the literature. All authors read and approved the final manuscript for submission.

\section{Acknowledgements}

We would like to thank $\mathrm{Dr}$ Wu $\mathrm{H}$ for reviewing the manuscript and constructive suggestions.

\section{References}

1. Abbas AK, Trotta E, D RS, Marson A, Bluestone JA. Revisiting IL-2: Biology and therapeutic prospects. Sci Immunol 2018; 3.

2. Alqathama A. BRAF in malignant melanoma progression and metastasis: potentials and challenges. Am J Cancer Res 2020; 10: 1103-1114.

3. Araghi M, Soerjomataram I, Jenkins M, Brierley J, Morris E, Bray F et al. Global trends in colorectal cancer mortality: projections to the year 2035. Int J Cancer 2019; 144: 2992-3000.

4. Bagati A, Kumar S, Jiang P, Pyrdol J, Zou AE, Godicelj A et al. Integrin av $\beta 6-T G F \beta-S O X 4$ Pathway Drives Immune Evasion in Triple-Negative Breast Cancer. Cancer Cell 2021; 39: 54-67.e59.

5. Batlle E, Massagué J. Transforming Growth Factor- $\beta$ Signaling in Immunity and Cancer. Immunity 2019; 50: 924-940.

6. Bidkhori G, Benfeitas R, Klevstig M, Zhang C, Nielsen J, Uhlen M et al. Metabolic network-based stratification of hepatocellular carcinoma reveals three distinct tumor subtypes. Proc Natl Acad Sci U S A 2018; 115: E11874-e11883.

7. Bugter JM, Fenderico N, Maurice MM. Mutations and mechanisms of WNT pathway tumour suppressors in cancer. Nat Rev Cancer 2021; 21: 5-21.

8. Cheng X, Jin Z, Ji X, Shen X, Feng H, Morgenlander W et al. ETS variant 5 promotes colorectal cancer angiogenesis by targeting platelet-derived growth factor BB. Int J Cancer 2019; 145: 179-191.

9. Ciardiello D, Vitiello PP, Cardone C, Martini G, Troiani T, Martinelli E et al. Immunotherapy of colorectal cancer: Challenges for therapeutic efficacy. Cancer Treat Rev 2019; 76: 22-32.

10. Clara JA, Monge $C$, Yang $Y$, Takebe $N$. Targeting signalling pathways and the immune microenvironment of cancer stem cells - a clinical update. Nat Rev Clin Oncol 2020; 17: 204-232.

11. Crayne CB, Albeituni S, Nichols KE, Cron RQ. The Immunology of Macrophage Activation Syndrome. Front Immunol 2019; 10: 119.

12. Crespo J, Sun H, Welling TH, Tian Z, Zou W. T cell anergy, exhaustion, senescence, and stemness in the tumor microenvironment. Curr Opin Immunol 2013; 25: 214-221. 
13. Damilakis E, Mavroudis D, Sfakianaki M, Souglakos J. Immunotherapy in Metastatic Colorectal Cancer: Could the Latest Developments Hold the Key to Improving Patient Survival? Cancers (Basel) 2020; 12.

14. David CJ, Massagué J. Contextual determinants of TGF $\beta$ action in development, immunity and cancer. Nat Rev Mol Cell Biol 2018; 19: 419-435.

15. Dekker E, Tanis PJ, Vleugels JLA, Kasi PM, Wallace MB. Colorectal cancer. Lancet 2019; 394: 14671480.

16. Dong $C$, Zhao G, Zhong M, Yue Y, Wu L, Xiong S. RNA sequencing and transcriptomal analysis of human monocyte to macrophage differentiation. Gene 2013; 519: 279-287.

17. Dumortier M, Ladam F, Damour I, Vacher S, Bièche I, Marchand N et al. ETV4 transcription factor and MMP13 metalloprotease are interplaying actors of breast tumorigenesis. Breast Cancer Res 2018; 20: 73.

18. Dy P, Wang W, Bhattaram P, Wang Q, Wang L, Ballock RT et al. Sox9 directs hypertrophic maturation and blocks osteoblast differentiation of growth plate chondrocytes. Dev Cell 2012; 22: 597-609.

19. Fang L, Miller YI. Regulation of lipid rafts, angiogenesis and inflammation by AIBP. Curr Opin Lipidol 2019; 30: 218-223.

20. Gautam S, Fioravanti J, Zhu W, Le Gall JB, Brohawn P, Lacey NE et al. The transcription factor c-Myb regulates CD8(+) T cell stemness and antitumor immunity. Nat Immunol 2019; 20: 337-349.

21. González-Silva L, Quevedo L, Varela I. Tumor Functional Heterogeneity Unraveled by scRNA-seq Technologies. Trends Cancer 2020; 6: 13-19.

22. Goossens P, Rodriguez-Vita J, Etzerodt A, Masse M, Rastoin O, Gouirand V et al. Membrane Cholesterol Efflux Drives Tumor-Associated Macrophage Reprogramming and Tumor Progression. Cell Metab 2019; 29: 1376-1389.e1374.

23. Grady WM, Myeroff LL, Swinler SE, Rajput A, Thiagalingam S, Lutterbaugh JD et al. Mutational inactivation of transforming growth factor beta receptor type II in microsatellite stable colon cancers. Cancer Res 1999; 59: 320-324.

24. Gwinn DM, Lee AG, Briones-Martin-Del-Campo M, Conn CS, Simpson DR, Scott Al et al. Oncogenic KRAS Regulates Amino Acid Homeostasis and Asparagine Biosynthesis via ATF4 and Alters Sensitivity to L-Asparaginase. Cancer Cell 2018; 33: 91-107.e106.

25. Ha Thi HT, Kim HY, Kim YM, Hong S. MicroRNA-130a modulates a radiosensitivity of rectal cancer by targeting SOX4. Neoplasia 2019; 21: 882-892.

26. Hardwick JC, Kodach LL, Offerhaus GJ, van den Brink GR. Bone morphogenetic protein signalling in colorectal cancer. Nat Rev Cancer 2008; 8: 806-812.

27. Harrison PT, Vyse S, Huang PH. Rare epidermal growth factor receptor (EGFR) mutations in nonsmall cell lung cancer. Semin Cancer Biol 2020; 61: 167-179.

28. Herzig S, Shaw RJ. AMPK: guardian of metabolism and mitochondrial homeostasis. Nat Rev Mol Cell Biol 2018; 19: 121-135. 
29. Hilger D, Masureel M, Kobilka BK. Structure and dynamics of GPCR signaling complexes. Nat Struct Mol Biol 2018; 25: 4-12.

30. Jinnouchi H, Guo L, Sakamoto A, Torii S, Sato Y, Cornelissen A et al. Diversity of macrophage phenotypes and responses in atherosclerosis. Cell Mol Life Sci 2020; 77: 1919-1932.

31. Katsuyama E, Yan M, Watanabe KS, Narazaki M, Matsushima S, Yamamura $Y$ et al. Downregulation of miR-200a-3p, Targeting CtBP2 Complex, Is Involved in the Hypoproduction of IL-2 in Systemic Lupus Erythematosus-Derived T Cells. J Immunol 2017; 198: 4268-4276.

32. Kobayashi T, Lam PY, Jiang H, Bednarska K, Gloury R, Murigneux V et al. Increased lipid metabolism impairs NK cell function and mediates adaptation to the lymphoma environment. Blood 2020; 136: 3004-3017.

33. Koh B, Hufford MM, Sun X, Kaplan MH. Etv5 Regulates IL-10 Production in Th Cells. J Immunol 2017; 198: 2165-2171.

34. Kurachi M. CD8(+) T cell exhaustion. Semin Immunopathol 2019; 41: 327-337.

35. Li H, Chen X, Gao Y, Wu J, Zeng F, Song F. XBP1 induces snail expression to promote epithelial- tomesenchymal transition and invasion of breast cancer cells. Cell Signal 2015; 27: 82-89.

36. Li T, Huang H, Shi G, Zhao L, Li T, Zhang Z et al. TGF- $\beta 1-S O X 9$ axis-inducible COL10A1 promotes invasion and metastasis in gastric cancer via epithelial-to-mesenchymal transition. Cell Death Dis 2018; 9: 849.

37. Liu C, Chikina M, Deshpande R, Menk AV, Wang T, Tabib T et al. Treg Cells Promote the SREBP1Dependent Metabolic Fitness of Tumor-Promoting Macrophages via Repression of CD8(+) T CellDerived Interferon-y. Immunity 2019; 51: 381-397.e386.

38. Meng ZZ, Liu W, Xia Y, Yin HM, Zhang CY, Su D et al. The pro-inflammatory signalling regulator Stat4 promotes vasculogenesis of great vessels derived from endothelial precursors. Nat Commun 2017; 8: 14640.

39. Min A, Jang H, Kim S, Lee KH, Kim DK, Suh KJ et al. Androgen Receptor Inhibitor Enhances the Antitumor Effect of PARP Inhibitor in Breast Cancer Cells by Modulating DNA Damage Response. Mol Cancer Ther 2018; 17: 2507-2518.

40. Moore KJ, Rosen ED, Fitzgerald ML, Randow F, Andersson LP, Altshuler D et al. The role of PPARgamma in macrophage differentiation and cholesterol uptake. Nat Med 2001; 7: 41-47.

41. Morimoto Y, Mizushima T, Wu X, Okuzaki D, Yokoyama Y, Inoue A et al. miR-4711-5p regulates cancer stemness and cell cycle progression via KLF5, MDM2 and TFDP1 in colon cancer cells. Br J Cancer 2020; 122: 1037-1049.

42. Morita K, Okamura T, Sumitomo S, Iwasaki Y, Fujio K, Yamamoto K. Emerging roles of Egr2 and Egr3 in the control of systemic autoimmunity. Rheumatology (Oxford) 2016; 55: ii76-ii81.

43. Musso G, Gambino R, Cassader M. Cholesterol metabolism and the pathogenesis of non-alcoholic steatohepatitis. Prog Lipid Res 2013; 52: 175-191. 
44. Oh DY, Bang YJ. HER2-targeted therapies - a role beyond breast cancer. Nat Rev Clin Oncol 2020; 17: 33-48.

45. Olingy CE, Dinh HQ, Hedrick CC. Monocyte heterogeneity and functions in cancer. J Leukoc Biol 2019; 106: 309-322.

46. Overman MJ, Lonardi S, Wong KYM, Lenz HJ, Gelsomino F, Aglietta M et al. Durable Clinical Benefit With Nivolumab Plus Ipilimumab in DNA Mismatch Repair-Deficient/Microsatellite Instability-High Metastatic Colorectal Cancer. J Clin Oncol 2018; 36: 773-779.

47. Papalexi E, Satija R. Single-cell RNA sequencing to explore immune cell heterogeneity. Nat Rev Immunol 2018; 18: 35-45.

48. Park JE, Dutta B, Tse SW, Gupta N, Tan CF, Low JK et al. Hypoxia-induced tumor exosomes promote M2-like macrophage polarization of infiltrating myeloid cells and microRNA-mediated metabolic shift. Oncogene 2019; 38: 5158-5173.

49. Park JS, Lee S, Jeong AL, Han S, Ka HI, Lim JS et al. Hypoxia-induced IL-32 $\beta$ increases glycolysis in breast cancer cells. Cancer Lett 2015; 356: 800-808.

50. Park Y, Reyna-Neyra A, Philippe L, Thoreen CC. mTORC1 Balances Cellular Amino Acid Supply with Demand for Protein Synthesis through Post-transcriptional Control of ATF4. Cell Rep 2017; 19: 10831090.

51. Sadik A, Somarribas Patterson LF, Öztürk S, Mohapatra SR, Panitz V, Secker PF et al. IL4I1 Is a Metabolic Immune Checkpoint that Activates the AHR and Promotes Tumor Progression. Cell 2020; 182: 1252-1270.e1234.

52. Scavo MP, Fucci L, Caldarola L, Mangia A, Azzariti A, Simone G et al. Frizzled-10 and cancer progression: Is it a new prognostic marker? Oncotarget 2018; 9: 824-830.

53. Schaefer KN, Peifer M. Wnt/Beta-Catenin Signaling Regulation and a Role for Biomolecular Condensates. Dev Cell 2019; 48: 429-444.

54. Selvarajah B, Azuelos I, Platé M, Guillotin D, Forty EJ, Contento G et al. mTORC1 amplifies the ATF4dependent de novo serine-glycine pathway to supply glycine during TGF- $\beta(1)$-induced collagen biosynthesis. Sci Signal 2019; 12.

55. Shimano H, Sato R. SREBP-regulated lipid metabolism: convergent physiology - divergent pathophysiology. Nat Rev Endocrinol 2017; 13: 710-730.

56. Sounni NE, Noel A. Targeting the tumor microenvironment for cancer therapy. Clin Chem 2013; 59: 85-93.

57. Sun Y, Shen S, Liu X, Tang H, Wang Z, Yu Z et al. MiR-429 inhibits cells growth and invasion and regulates EMT-related marker genes by targeting Onecut2 in colorectal carcinoma. Mol Cell Biochem 2014; 390: 19-30.

58. Sung H, Ferlay J, Siegel RL, Laversanne M, Soerjomataram I, Jemal A et al. Global Cancer Statistics 2020: GLOBOCAN Estimates of Incidence and Mortality Worldwide for 36 Cancers in 185 Countries. CA Cancer J Clin 2021; 71: 209-249. 
59. Szebeni GJ, Vizler C, Nagy LI, Kitajka K, Puskas LG. Pro-Tumoral Inflammatory Myeloid Cells as Emerging Therapeutic Targets. Int J Mol Sci 2016; 17.

60. Talty R, Olino K. Metabolism of Innate Immune Cells in Cancer. Cancers (Basel) 2021; 13.

61. Turnham DJ, Yang WW, Davies J, Varnava A, Ridley AJ, Conlan RS et al. Bcl-3 promotes multi-modal tumour cell migration via NF-KB1 mediated regulation of Cdc42. Carcinogenesis 2020; 41: 14321443.

62. van der Leun AM, Thommen DS, Schumacher TN. CD8(+) T cell states in human cancer: insights from single-cell analysis. Nat Rev Cancer 2020; 20: 218-232.

63. Veremeyko T, Yung AWY, Anthony DC, Strekalova T, Ponomarev ED. Early Growth Response Gene-2 Is Essential for M1 and M2 Macrophage Activation and Plasticity by Modulation of the Transcription Factor CEBPß. Front Immunol 2018; 9: 2515.

64. Vergara D, Stanca E, Guerra F, Priore P, Gaballo A, Franck J et al. $\beta$-Catenin Knockdown Affects Mitochondrial Biogenesis and Lipid Metabolism in Breast Cancer Cells. Front Physiol 2017; 8: 544.

65. Wang Y, Ding X, Liu B, Li M, Chang Y, Shen H et al. ETV4 overexpression promotes progression of non-small cell lung cancer by upregulating PXN and MMP1 transcriptionally. Mol Carcinog 2020; 59: 73-86.

66. Wherry EJ, Kurachi M. Molecular and cellular insights into T cell exhaustion. Nat Rev Immunol 2015; 15: 486-499.

67. Xu M, Pokrovskii M, Ding Y, Yi R, Au C, Harrison OJ et al. c-MAF-dependent regulatory T cells mediate immunological tolerance to a gut pathobiont. Nature 2018; 554: 373-377.

68. Yaghoubi N, Soltani A, Ghazvini K, Hassanian SM, Hashemy SI. PD-1/ PD-L1 blockade as a novel treatment for colorectal cancer. Biomed Pharmacother 2019; 110: 312-318.

69. Yuan P, Yang T, Mu J, Zhao J, Yang Y, Yan Z et al. Circadian clock gene NPAS2 promotes reprogramming of glucose metabolism in hepatocellular carcinoma cells. Cancer Lett 2020; 469: 498-509.

70. Yuan X, Dong D, Li Z, Wu B. Rev-erba activation down-regulates hepatic Pck1 enzyme to lower plasma glucose in mice. Pharmacol Res 2019; 141: 310-318.

71. Zeng L, Xiao Q, Chen M, Margariti A, Martin D, Ivetic A et al. Vascular endothelial cell growth-activated XBP1 splicing in endothelial cells is crucial for angiogenesis. Circulation 2013; 127: 1712-1722.

72. Zhang L, Li ZY, Skrzypczynska KM, Fang Q, Zhang W, O'Brien SA et al. Single-Cell Analyses Inform Mechanisms of Myeloid-Targeted Therapies in Colon Cancer. Cell 2020; 181: 442-+.

73. Zhang M, Yan Y, Lim YB, Tang D, Xie R, Chen A et al. BMP-2 modulates beta-catenin signaling through stimulation of Lrp5 expression and inhibition of beta-TrCP expression in osteoblasts. J Cell Biochem 2009; 108: 896-905.

74. Zhang X, Wang Y, Liu X, Zhao A, Yang Z, Kong F et al. KIF2A promotes the progression via AKT signaling pathway and is upregulated by transcription factor ETV4 in human gastric cancer. Biomed Pharmacother 2020; 125: 109840. 
75. Zhang Y, Du W, Chen Z, Xiang C. Upregulation of PD-L1 by SPP1 mediates macrophage polarization and facilitates immune escape in lung adenocarcinoma. Exp Cell Res 2017; 359: 449-457.

76. Zheng L, Li L, Lu Y, Jiang F, Yang XA. SREBP2 contributes to cisplatin resistance in ovarian cancer cells. Exp Biol Med (Maywood) 2018; 243: 655-662.

77. Zhou Q, Lavorgna A, Bowman M, Hiscott J, Harhaj EW. Aryl Hydrocarbon Receptor Interacting Protein Targets IRF7 to Suppress Antiviral Signaling and the Induction of Type I Interferon. J Biol Chem 2015; 290: 14729-14739.

78. QJ, Lavorgna A, Bowman M, Hiscott J, Harhaj EW. Aryl Hydrocarbon Receptor Interacting Protein Targets IRF7 to Suppress Antiviral Signaling and the Induction of Type I Interferon. J Biol Chem 2015; 290: 14729-14739.

\section{Supplementary Table}

Supplementary Table 1 is not available with this version.

\section{Figures}



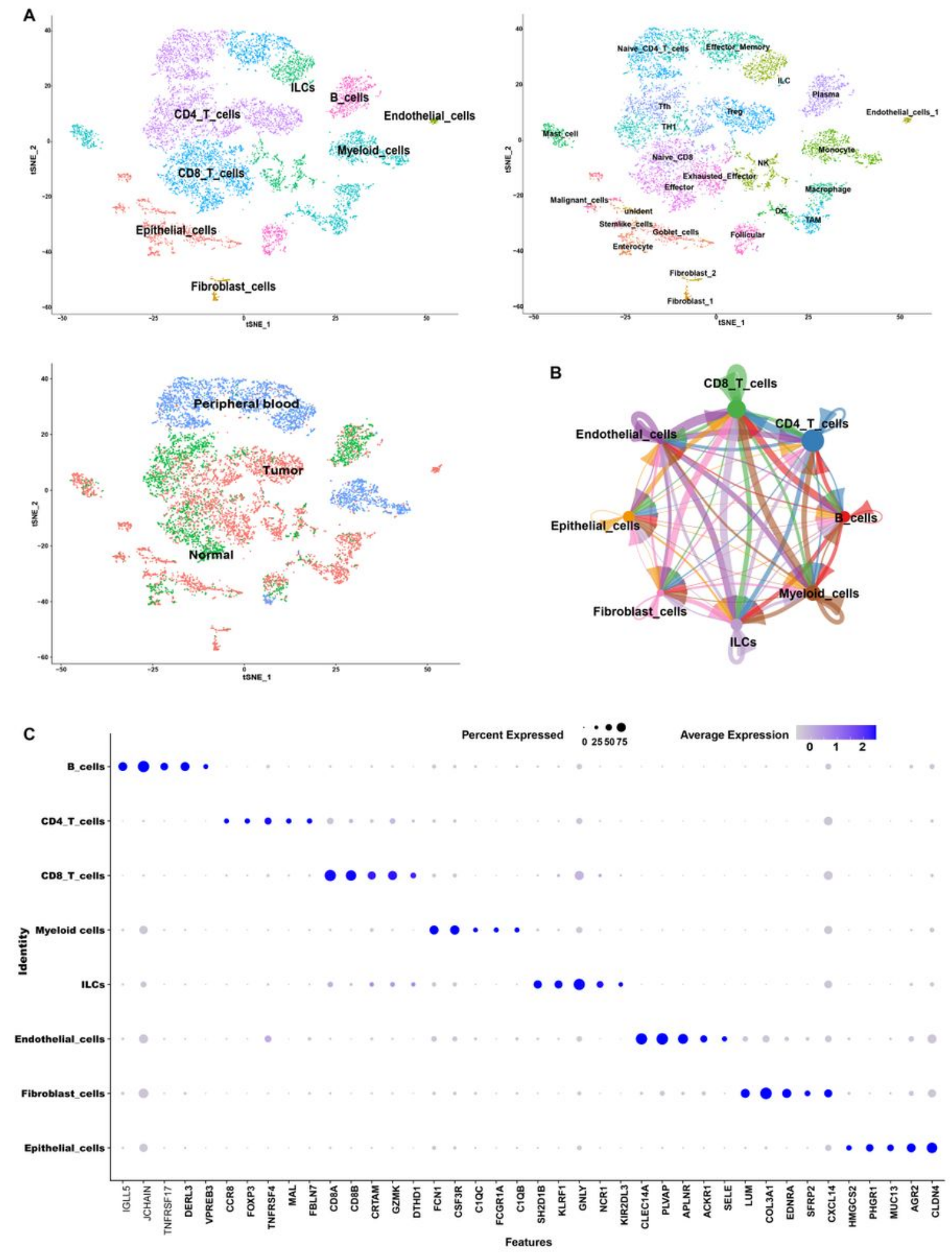

\section{Figure 1}

Overview of single cells derived from tumors, adjacent tumor tissues, and peripheral blood of CRC patients. (A-C) tSNE plots of all the single cells color-coded for (A) eight major cell types, (B) 25 sub-cell types, (C) tissue origins (tumor, adjacent to tumor or blood). (D) Interaction network among major cell types constructed by CellChat; circle sizes represent interaction weights; the thicker line indicates more 
weight and strength of the interactions between variable major cell types. (E) Top five marker genes of eight major cell types identified in this profile.
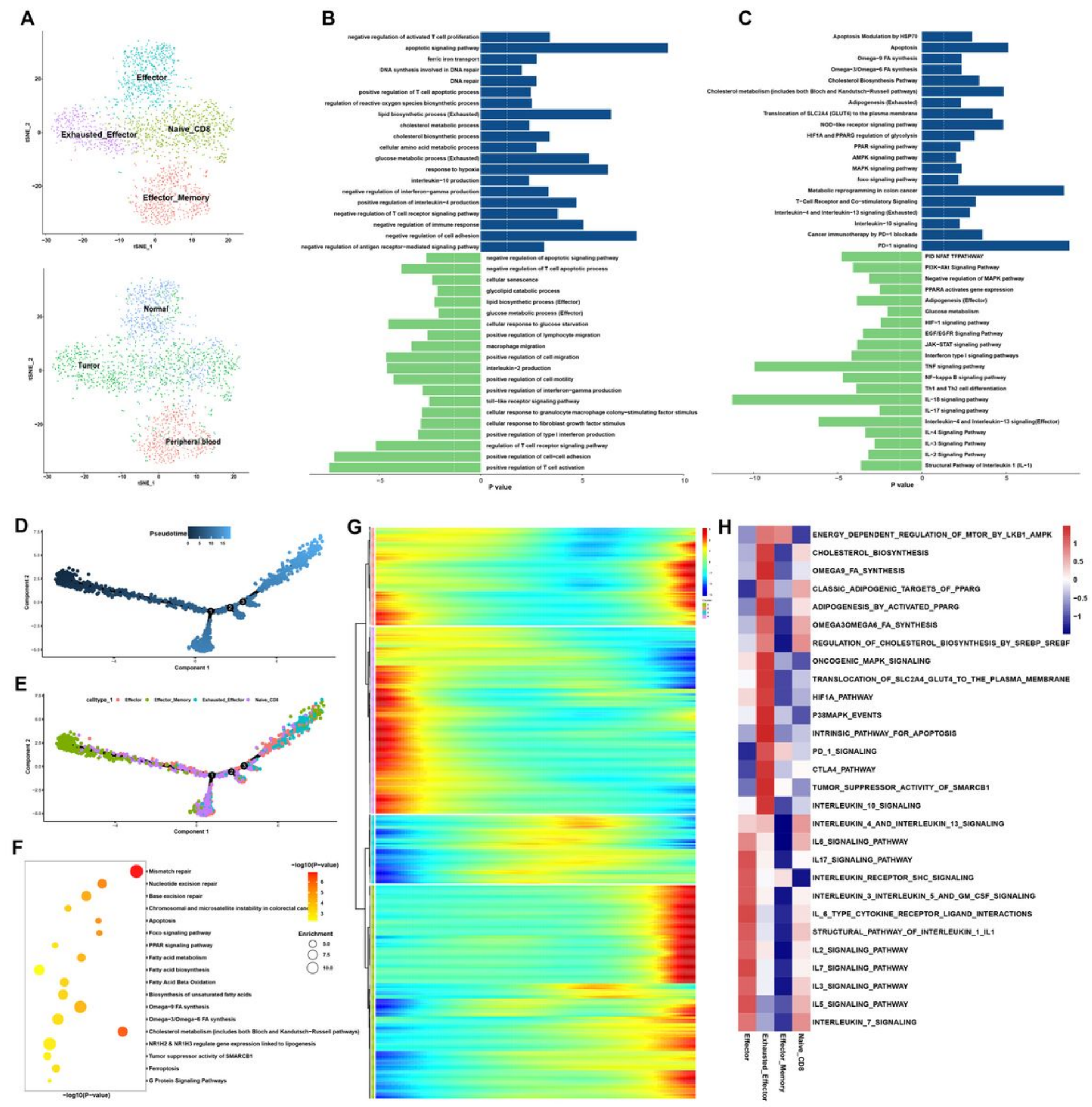

Figure 2

CD8+ T cells tend to exhaust in the tumor microenvironment. (A) tSNE plots of four sub-cell types of CD8+ T cells. (B) The tissue origins (tumor, adjacent to tumor or blood). (C-D) Enrichment analysis of marker genes of Tex and Teff. The height of each barplot shows the log10 of P-value calculated using the Metascape website. (E-F) Differentiation trajectory of CD8+ T cells in CRC, color-coded for pseudotime 
(E) and sub-cell types (F). (G-H) Pseudo-heatmap of genes altered in the differentiation process of CD8+ $T$ cells in CRC, divided into four clusters. The bubble plot shows the enrichment functions and pathways of genes in cluster 1 according to Metascape. (I) Differences between "c2.all.v7.4.symbols.gmt" and "c5.all.v7.4.symbols.gmt" scored by GSVA. The mean expression level for functions or pathways was transformed into a row Z score.

A .

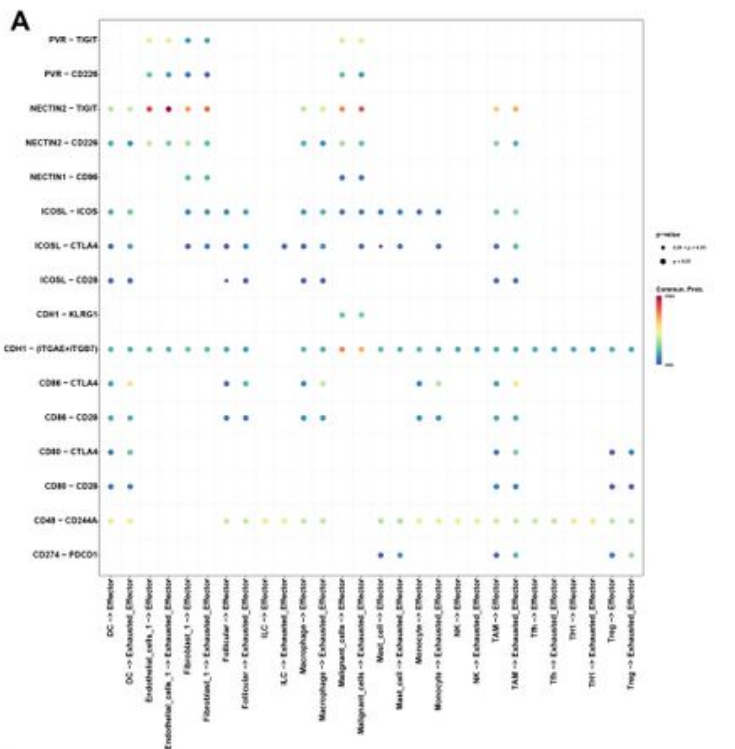

C
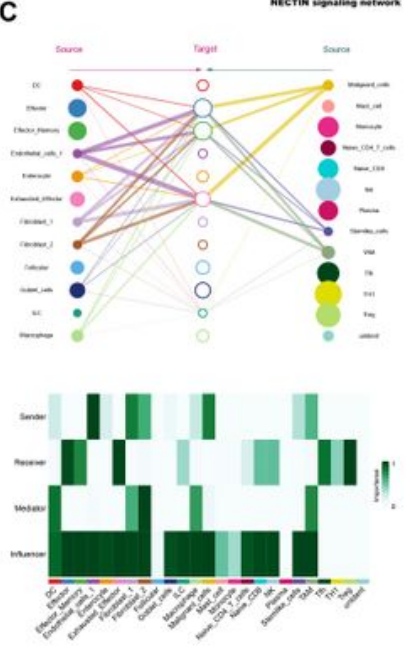

E

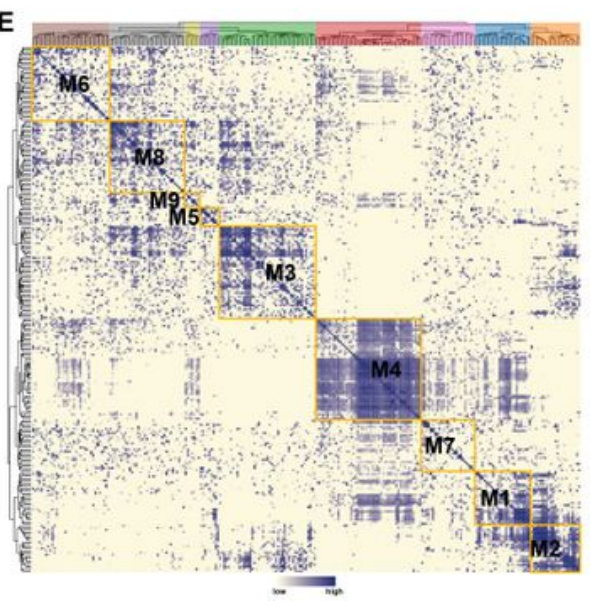

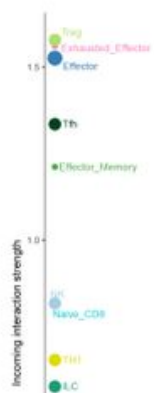

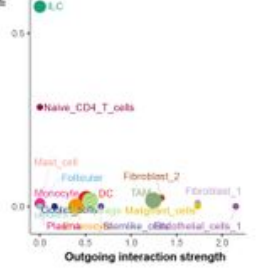

B

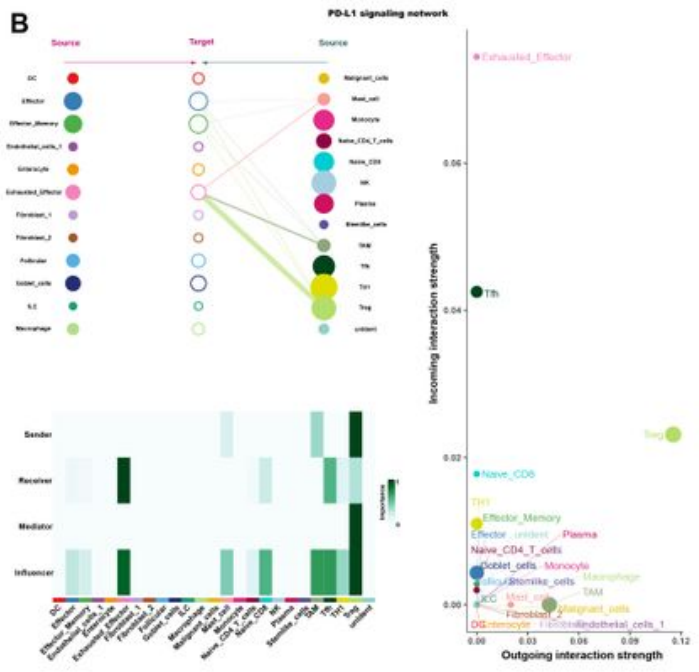

D
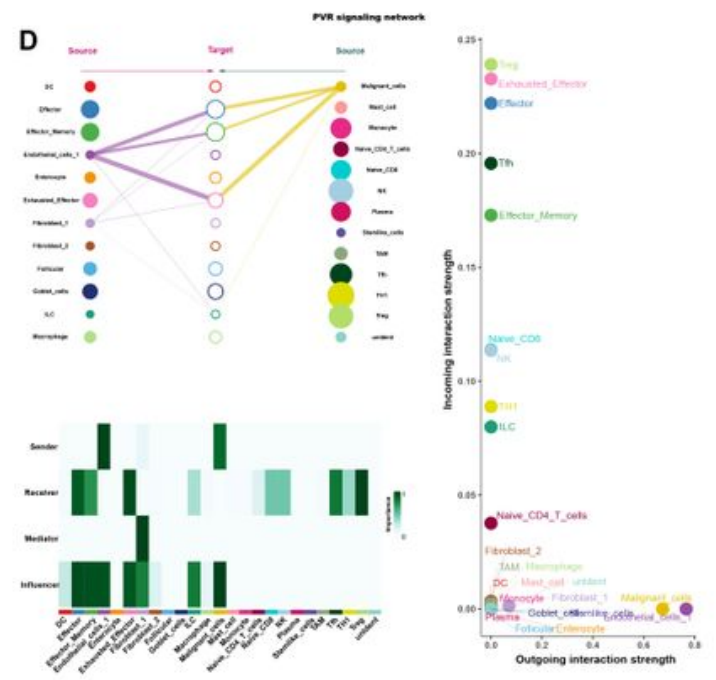
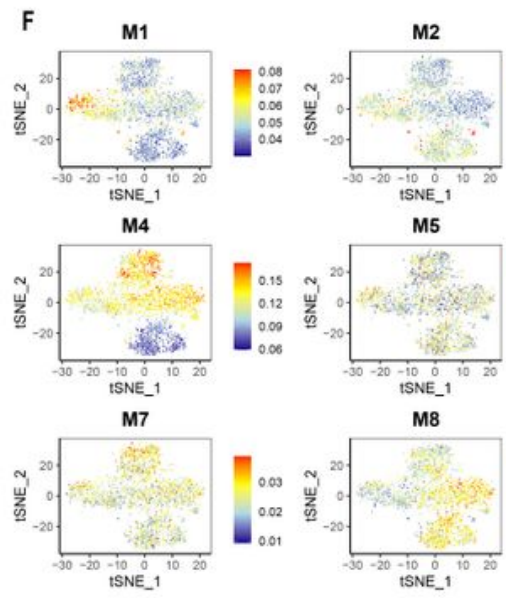
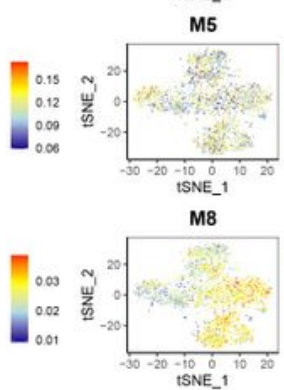
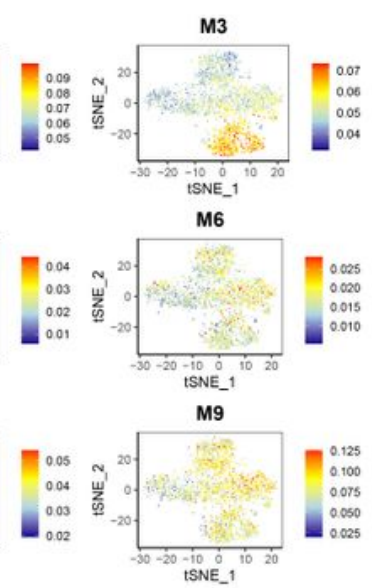

Figure 3 
The interaction network and transcription regulatory network of CD8+ T cells. (A) Ligand-receptor interactions between CD8+ T cells and TME-infiltrated cell types detected by CellChat. The colors and sizes in the bubble plot represent the communication probability, and P-values of the ligand-receptor pairs; blue and red colors correspond to the smallest and largest values, respectively. (B-D) Hierarchical plot, bubble plot, and heatmap showing the inferred intercellular communication networks for PD-L1 (B), NECTIN2 (C), and PVR (D) signaling, respectively. The circle sizes in the hierarchical plot are proportional to the number of cells of each cell type, and the edge width represents the communication probability. The heatmap shows the relative importance of each cell type based on four computed network centrality measures for each signaling network. The bubble plot shows the dominant senders (sources) and receivers (targets) of each signaling type. (E) Heatmap of identified regulon modules based on the regulon CSI matrix. (F) t-SNE map for all CD8+ T cells based on the regulon activity scores (RAS) of the regulon modules. 

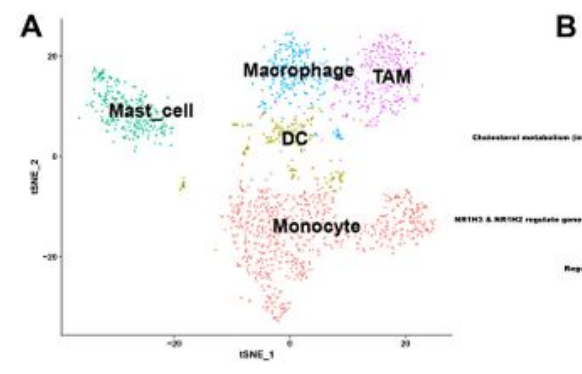

B

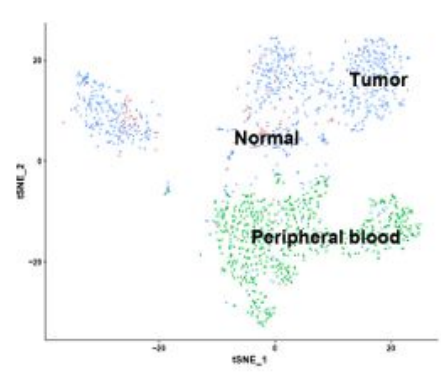

D
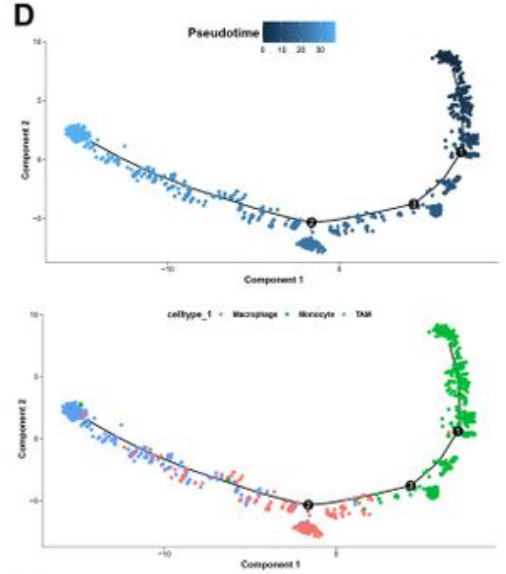

E

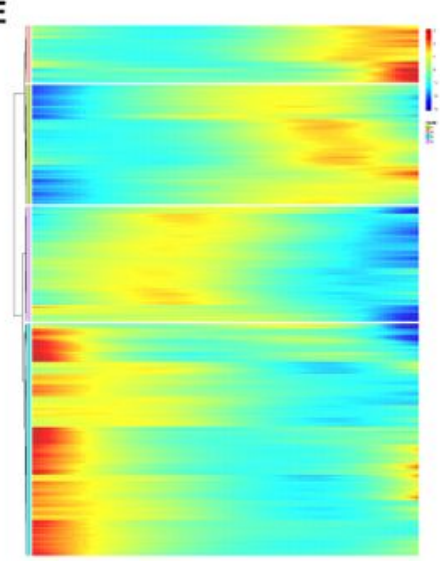

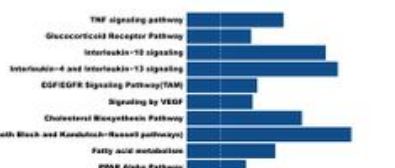
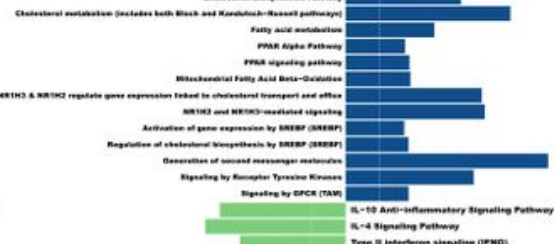

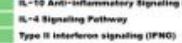

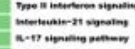
art

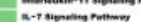

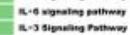

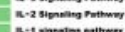

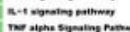

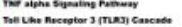

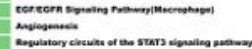

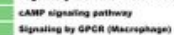

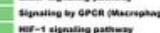
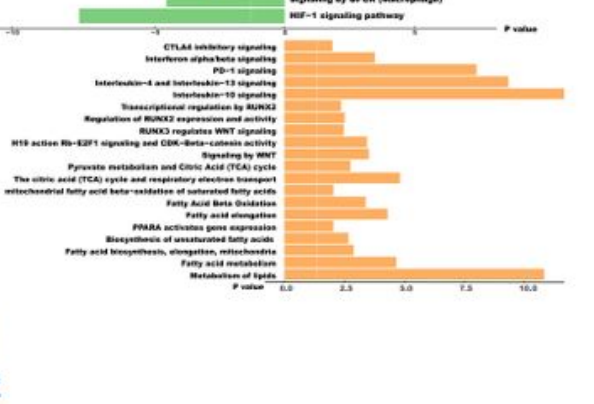

F

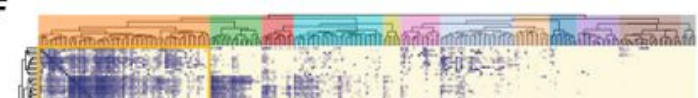

M2
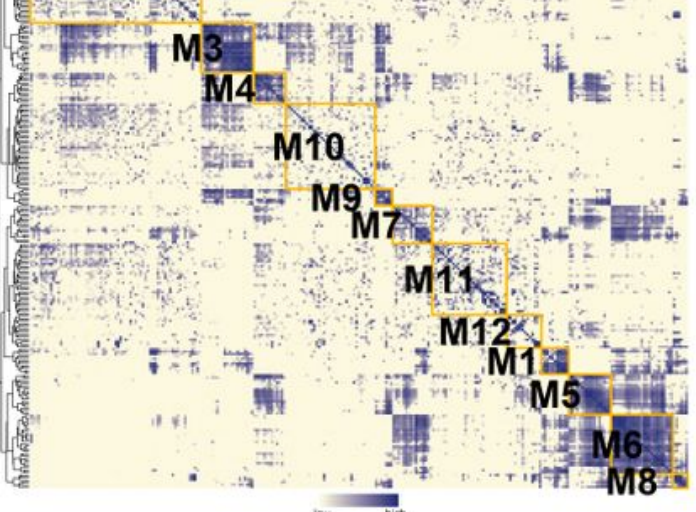

(1)

G

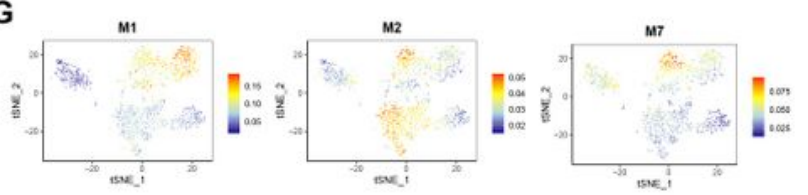

\section{Figure 4}

Macrophages resulted from M1 polarization, whereas TAMs resulted from M2 polarization in CRC. (A) tSNE plot of four sub-cell types of myeloid cells. (B) The tissue origins (tumor, adjacent to tumor or blood). (C) Enrichment analysis of marker genes of TAMs (blue), macrophages (green), and DCs (orange). The height of each barplot shows the log10 P-value calculated by Metascape. (D) Differences in "c2.all.v7.4.symbols.gmt" and "c5.all.v7.4.symbols.gmt" scored by GSVA. The mean expression level of 
functions or pathways was transformed into a row $Z$ score. (E-F) Differentiation trajectory of monocytes, macrophages, and TAMs in CRC, color-coded for pseudotime (E), and sub-cell types (F). (G) Pseudoheatmap of genes altered in the differentiation process of monocytes, macrophages, and TAMs in CRC, grouped into four clusters.
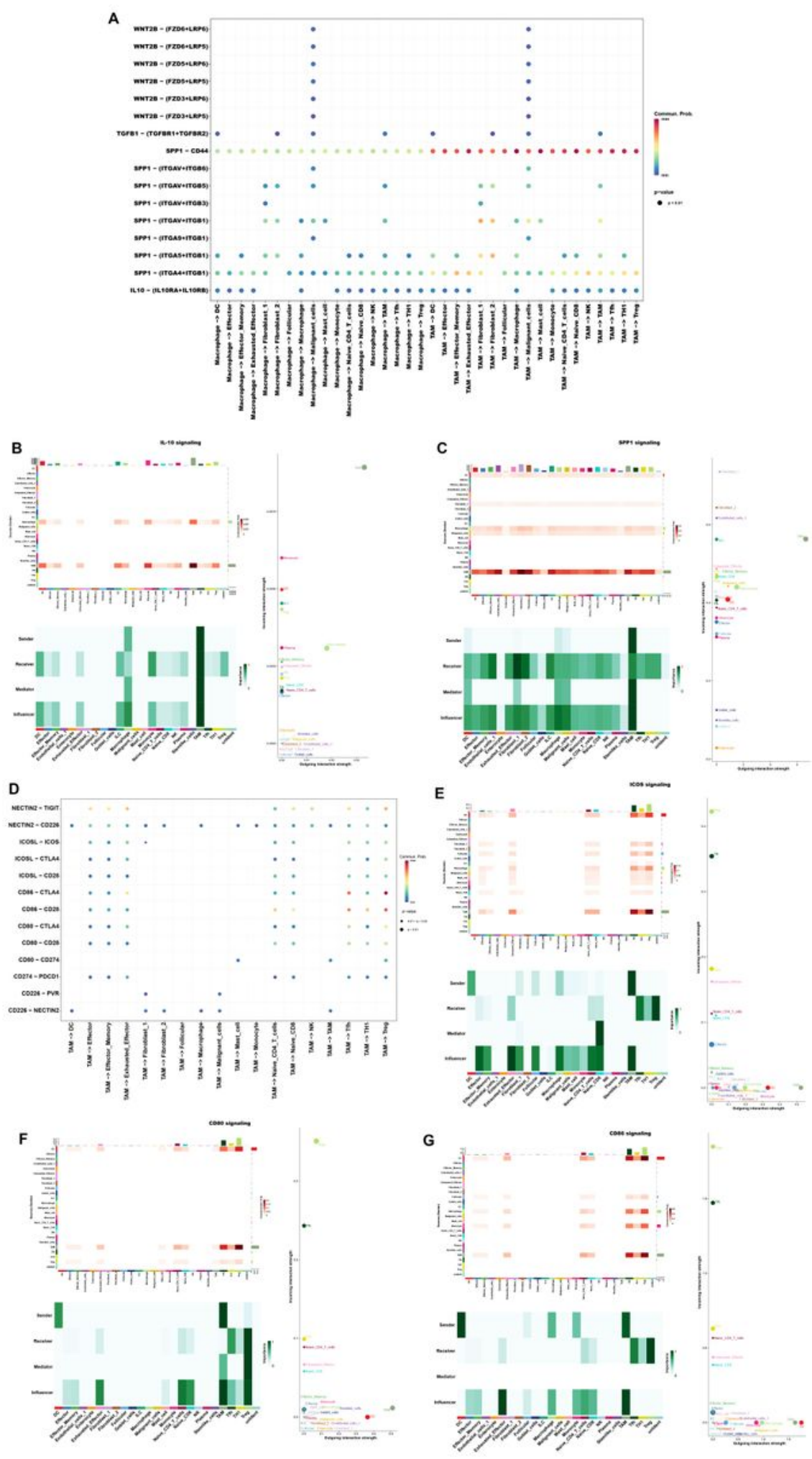

Figure 5 
The interaction network of macrophages and TAMs. (A) Soluble factor interactions among macrophages, TAMs, and TME-infiltrated cell types. (G) Ligand-receptor interactions of immune checkpoints between TAMs and TME-infiltrated cell clusters detected by CellChat. Selected ligand-receptor pairs are shown in the bubble plot. The colors and sizes in the bubble plot represent the communication probability, and Pvalues of the ligand-receptor pairs; blue and red colors correspond to the smallest and largest values, respectively. (B-C, E-G) Hierarchical plot, bubble plot, and heatmap showing the inferred intercellular communication network for SPP1 (B), IL-10 (C), CD80 (E), CD86 (F), and ICOSL (G) signaling. The circle sizes in the hierarchical plot are proportional to the number of cells in each cell type, and the edge width represents the communication probability. The heatmap shows the relative importance of each cell type based on the four computed network centrality measures of each signaling network. The bubble plot shows the dominant senders (sources) and receivers (targets) of each signaling type.

A

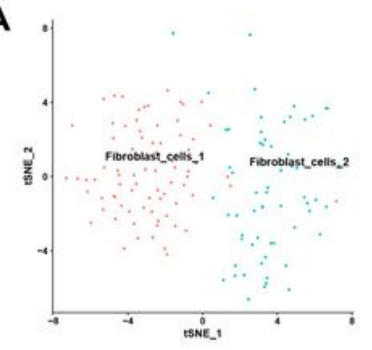

B

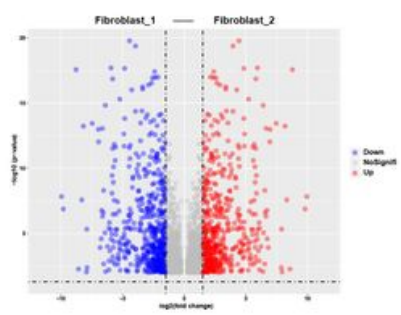

E

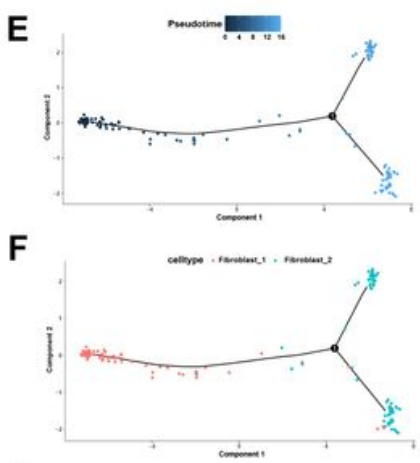

G

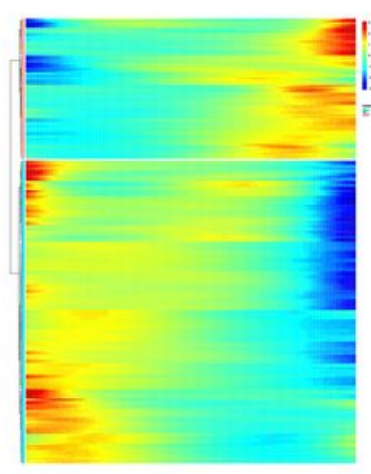

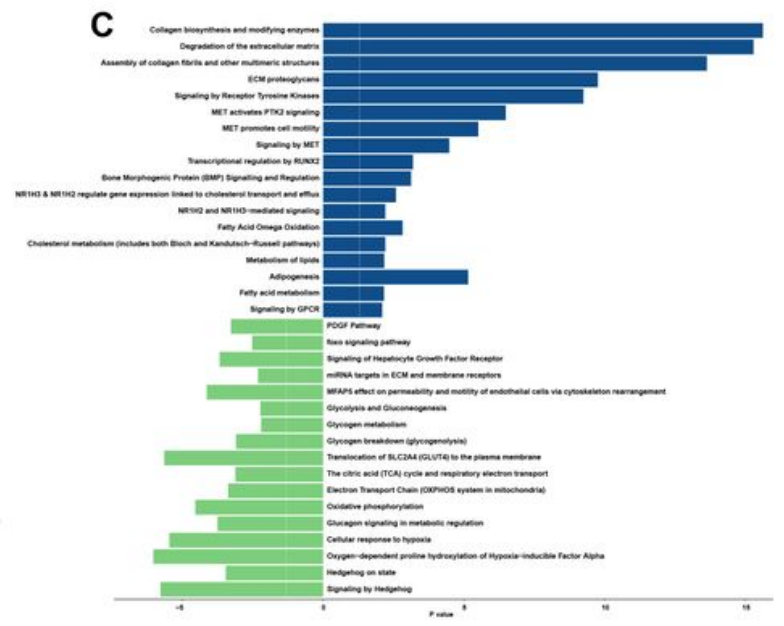

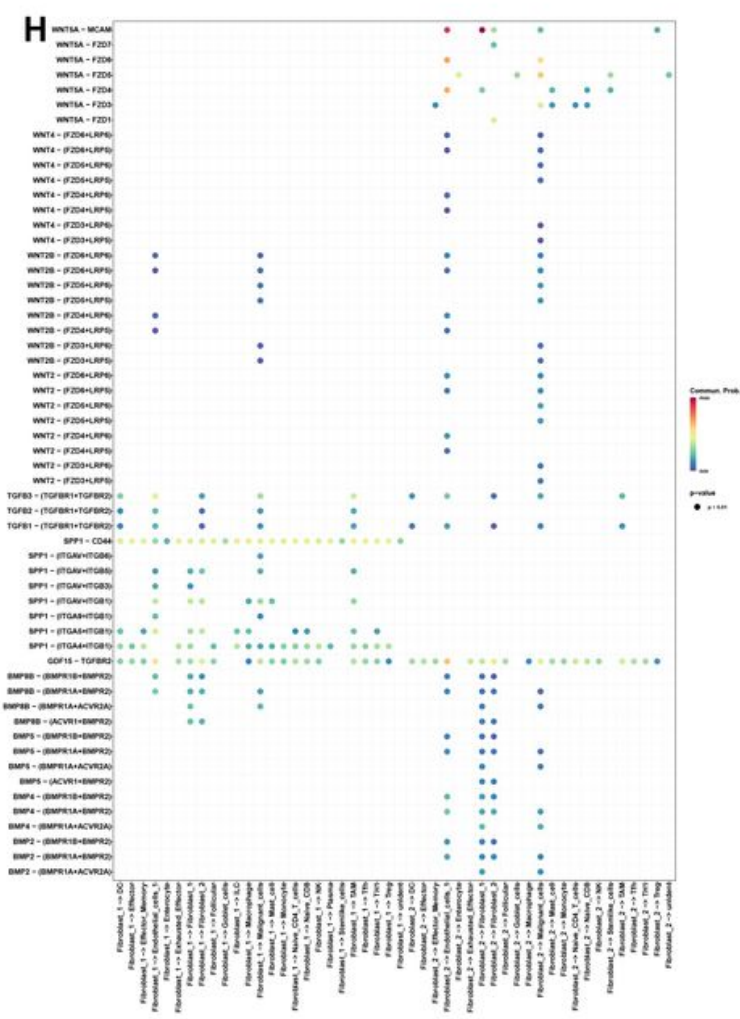

D

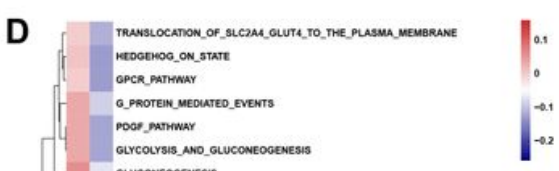

$-20$

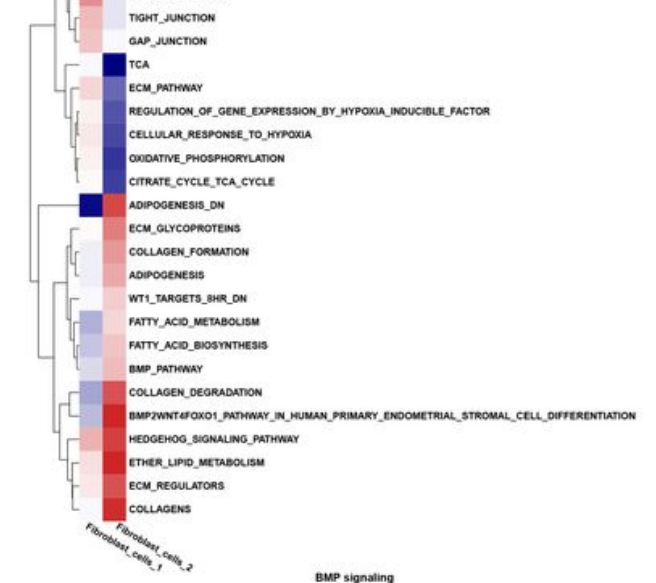

I
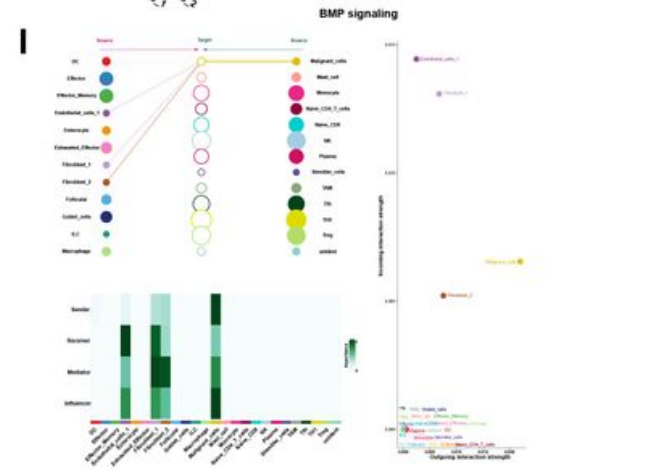

J

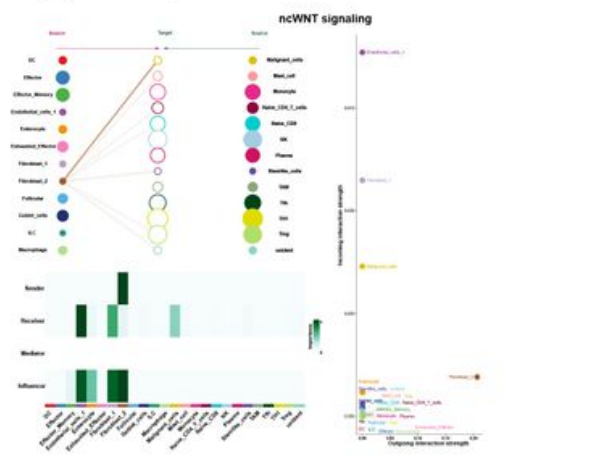

Figure 6 
Hypoxia and acid signals promote metabolic and functional remodeling in fibroblasts. (A) tSNE plot of two sub-groups of fibroblasts. (B) Volcano plot of DEGs between fibroblast-1 and fibroblast-2. (C) Enrichment analysis of DEGs of fibroblast-2 (blue) and fibroblast-1 (green). The height of each barplot shows the log10 P-value calculated by Metascape. (D) Differences in "c2.all.v7.4.symbols.gmt" and "c5.all.v7.4.symbols.gmt" scored by GSVA. (E-F) Differentiation trajectories of monocytes, macrophages, and TAMs in CRC, color-coded for pseudotime (E) and sub-cell types (F). (G) Pseudo-heatmap of genes altered in the differentiation process of monocytes, macrophages, and TAMs in CRC, divided into four clusters. $(\mathrm{H})$ Ligand-receptor interactions between fibroblasts and TME-infiltrated cell types detected by CellChat. The colors and sizes in the bubble plot represent the communication probabilities, and P-values of the ligand-receptor pairs; blue and red colors correspond to the smallest and largest values, respectively. (I-J) Hierarchical plot, bubble plot, and heatmap showing the inferred intercellular communication network for ncWNT (I) and BMP $(\mathrm{J})$ signaling. 

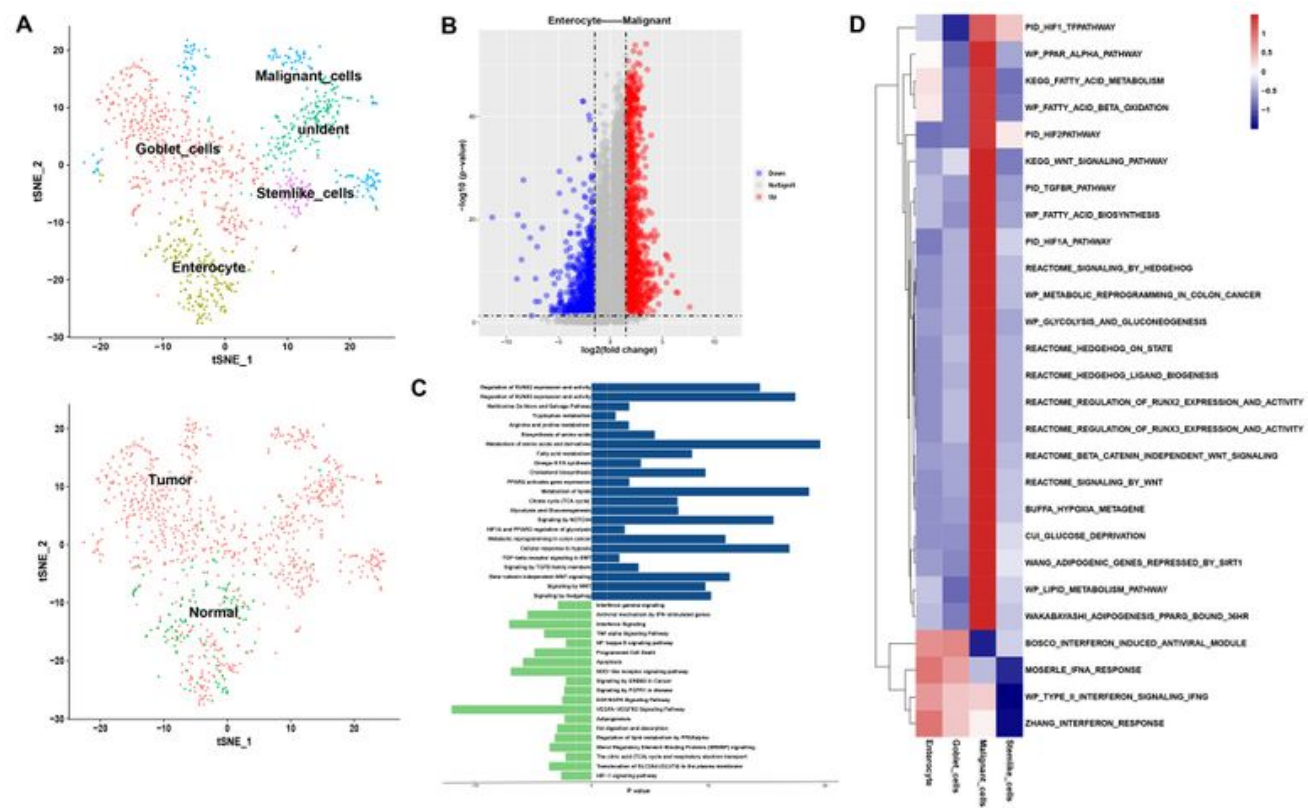

E

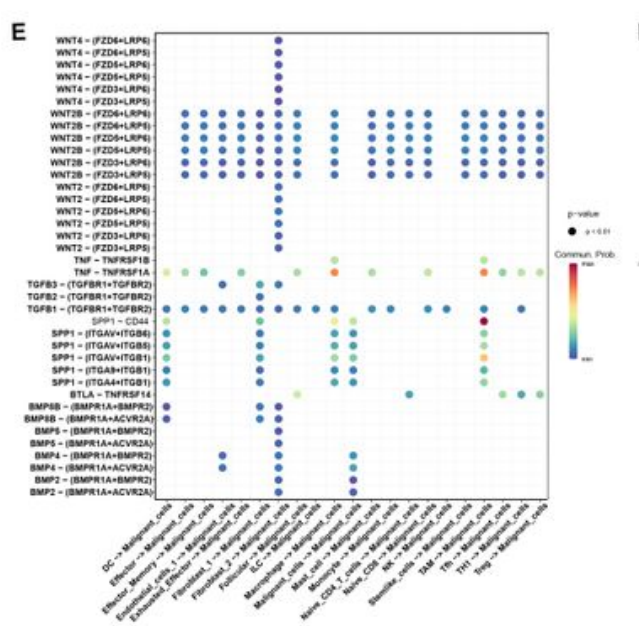

F

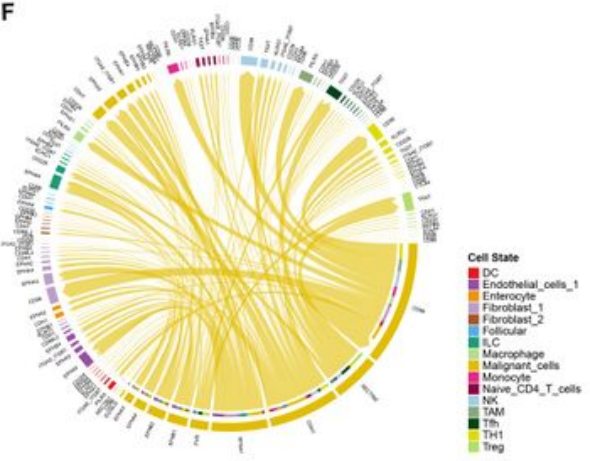

G
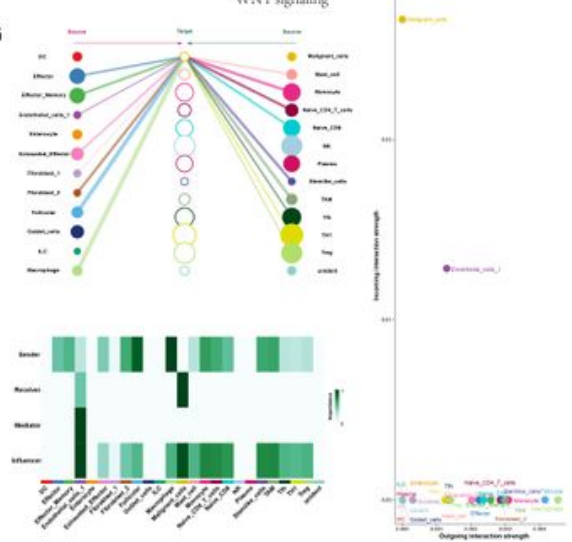
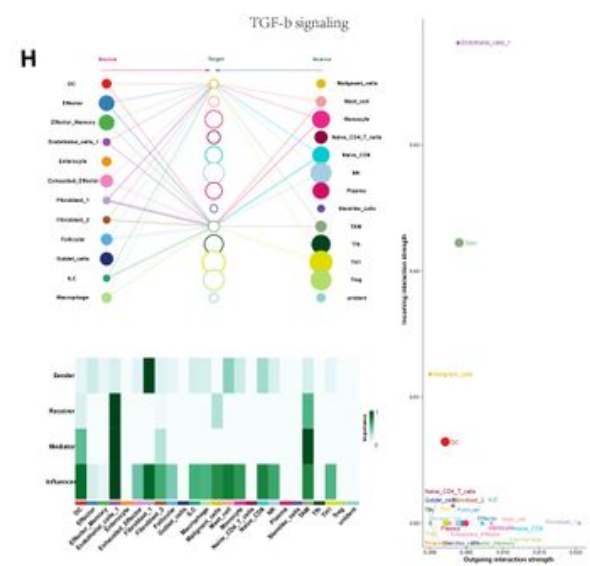

Figure 7

CRC cells engaged in the construction of the immune-suppressive microenvironment. (A) tSNE plot of two sub-groups of epithelial cells. (B) The tissue origins (tumor, adjacent to tumor or blood). (C) Volcano plot of DEGs between enterocytes and malignant cells. (D) Enrichment analysis of DEGs of malignant cells (blue) and enterocytes (green). The height of each barplot shows the log10 P-value calculated by Metascape. (D) Differences in "c2.all.v7.4.symbols.gmt" and "c5.all.v7.4.symbols.gmt" scored by GSVA. 
(E-F) Soluble factors and ligand-receptor interactions between malignant cells and TME-infiltrated cell types detected by CellChat. The colors and sizes in the bubble plot represent the communication probabilities, and P-values of the ligand-receptor pairs; blue and red colors correspond to the smallest and largest values, respectively. $(\mathrm{G}-\mathrm{H})$ Hierarchical plot, bubble plot, and heatmap showing the inferred intercellular communication network for Wnt $(\mathrm{G})$ and SPP1 $(\mathrm{H})$ signaling.
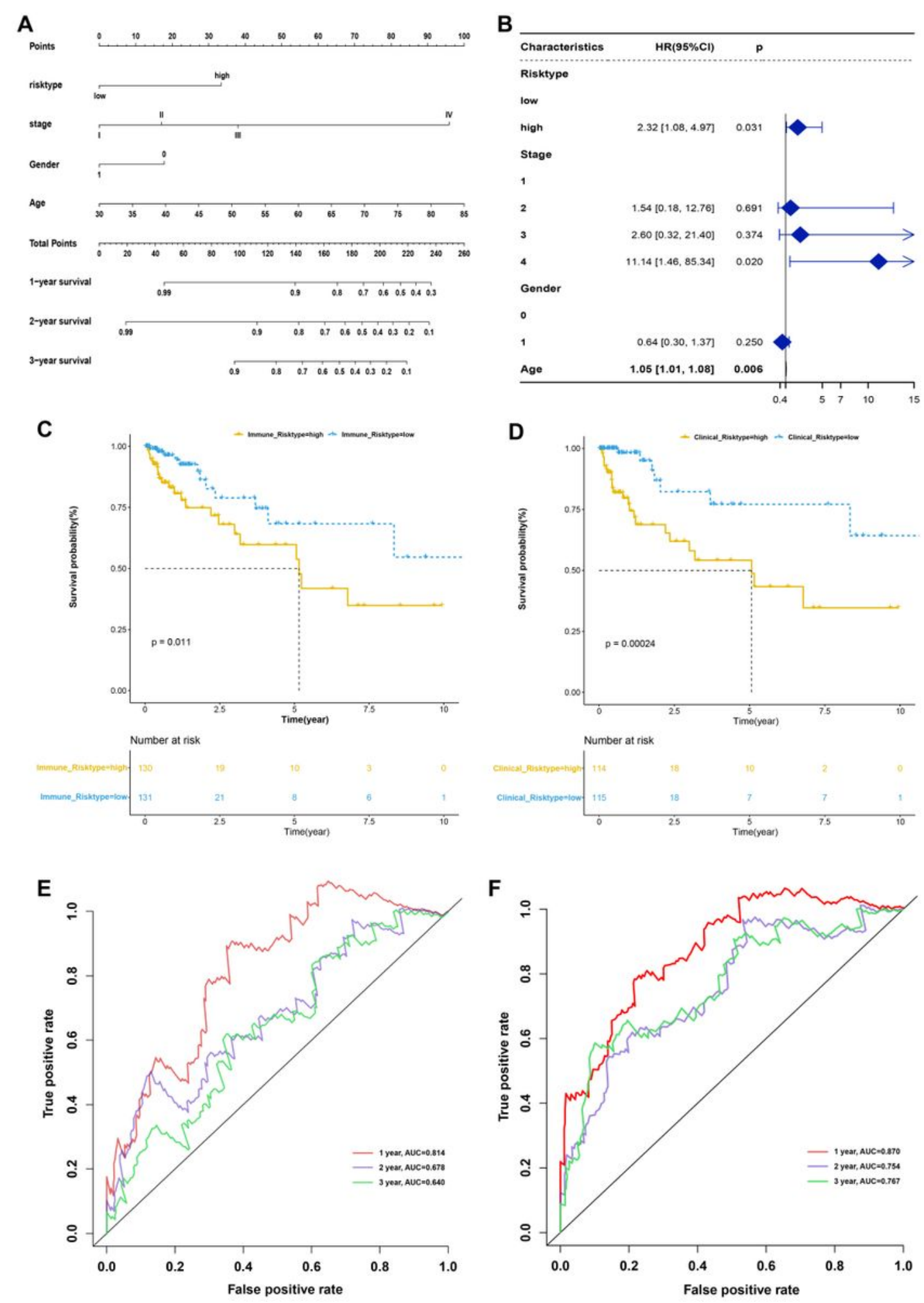

Figure 8 
Infiltration of tumor-educated immune cells is associated with a worse prognosis in CRC. (A) Nomogram of independent prognostic factors including risktype, TNM stage, gender, and age. The values attributed to each patient are located on the variable axis; the upward line shows the points received for each variable. The sum of the scores is shown on the total points axis; the line drawn downward to the survival axis shows the likelihood of 1-, 3-, or 5-year survival. (B) Forest plot showing the hazard ratio and P-value of each prognostic factor. (C-D) KM survival curves for immune risktype and clinical risktype. (E-F) Immune risk scores and clinical risk scores determined by time-dependent ROC curves. The areas under the ROC curve were $0.814,0.678$, and 0.640 for the immune risk model for $1-, 3$-, and 5 -year OS versus $0.870,0.754$, and 0.767 for the clinical risk model for $1-, 3$-, and 5 -year OS, respectively.

\section{Supplementary Files}

This is a list of supplementary files associated with this preprint. Click to download.

- SupplementaryFile.docx 